\title{
Nuclear Cycler: An Incremental Approach to the Deflection of Asteroids
}

\author{
Massimiliano Vasile ${ }^{1}$, Nicolas Thiry ${ }^{2, *}$ \\ 75 Montrose Street, Glasgow, UK G1 $1 X Q$
}

\begin{abstract}
This paper introduces a novel deflection approach based on nuclear explosions: the nuclear cycler. The idea is to combine the effectiveness of nuclear explosions with the controllability and redundancy offered by slow push methods within an incremental deflection strategy. The paper will present an extended model for single nuclear stand-off explosions in the proximity of elongated ellipsoidal asteroids, and a family of natural formation orbits that allows the spacecraft to deploy multiple bombs while being shielded by the asteroid during the detonation.
\end{abstract}

Keywords: Asteroid deflection; planetary defence; nuclear interceptor

\section{Introduction}

Deflection methods are commonly divided into two main categories, impulsive and slow push, depending on whether the modification of the orbit of the asteroid is, respectively, quasi-instantaneous or modified over a longer period of time. Examples of impulsive methods include the nuclear interceptor (Hammerling and Remo , 1995) and the kinetic impactor (Jutzia and Michel , 2014), while slow-push methods include, among others, the gravity tractor (Lu and Love , 2005), laser ablation (Vasile and Maddock,

\footnotetext{
*Corresponding author

Email addresses: massimiliano.vasile@strath.ac.uk (Massimiliano Vasile), nicolas.thiry@strath.ac.uk (Nicolas Thiry)

${ }^{1}$ Professor at the University of Strathclyde

${ }^{2} \mathrm{PhD}$ Candidate at the University of Strathclyde
} 
2012), ion-beam shepherd (Bombardelli and Pel?ez , 2011) and mass driver (Olds, Charania, Graham and Wallace, 2004).

The nuclear interceptor can nudge the asteroid off of its collision course with the Earth even when the warning time is low, but a single explosion represents a single point of failure and does not allow any control over the evolution of the trajectory of the asteroid. On the other hand, slowpush methods allow for a more precise control of the deflection manoeuvre but typically require a longer warning time, additional propellant in order to maintain a hovering position in the vicinity of the asteroid, the ability to operate autonomously and are dependent on the distance from the Sun (Bombardelli and Pel?ez, 2011; Sanchez, Colombo, Vasile et al. , 2009; Vasile and Maddock, 2012).

Nuclear methods carry the highest energy density among all currently proposed mitigation strategies. As there is no atmosphere in space, the efficiency of nuclear methods is based on the amount of asteroid material that can be blasted away following the explosion. In a report to Congress, NASA (2007) argued that using a stand-off nuclear detonation would be ten to a hundred times more effective than any other alternative. While a subterranean explosion would, in principle, further increase the amount of material that can be expelled, a stand-off configuration does not require landing and digging and is thus more manageable with current technology.

The theoretical efficiency of nuclear-based approaches must be balanced with the difficulty in controlling the outcome of the explosion. This lack of control can lead to three main problems. The high level of energy released during the single detonation introduces the potential risk of an unwanted fragmentation. If the asteroid breaks up into several pieces following the explosion, it may be that some of the larger pieces will impact the Earth and the probability of causing damages may never go to zero (Sanchez, Vasile and Radice , 2010); the risk of fragmentation is already reduced however due to the choice of the stand-off configuration.

Another problem arises from the precise detonation at the required location. Choosing such a location could actually require the addition of an observer spacecraft, as it is the case for the kinetic impactor. Last but not least, the current epistemic uncertainty on the properties of the asteroid translates into a significant variance on the expected deflection. In particular, as will be shown in this paper, the efficiency of the nuclear interceptor relies strongly on the amount of energy required to vaporize the asteroid material, which itself is not so well characterized in the available literature. 
Hence, relying on a single interceptor is a rather risky strategy.

The idea proposed in this paper partially overcomes these difficulties by fractionating a single explosion into a number of smaller and better controlled ones. A single spacecraft, carrying a number of bombs, is placed on a formation orbit with the asteroid and incrementally releases the bombs so that each of them explodes at an optimal position with respect to the surface of the asteroid. As will be shown, careful choices in the firing time and orbital trajectory can allow for the incremental deflection of the asteroid while ensuring an appropriate radiation shielding to the carrier.

The paper is structured as follows: first by a review of the model of a single nuclear interceptor method considering both spherical and elongated asteroids. The idea of the nuclear cycler is then explained, illustrating the concept with a possible choice of mission configuration. The following section shows the results of a comparison for the deflection of an elongated Apophislike asteroid using a single interceptor and an incremental deflection using the nuclear cycler idea. Lastly is a discussion on the strategy and plans for future work.

\section{Single Detonator Model}

This section introduces a model to calculate the change in linear momentum of the asteroid due to a stand-off nuclear explosion. The first subsection presents a slightly modified version of the model presented by Sanchez, Colombo, Vasile et al. (2009) applicable to the case of a spherical asteroid. The model is then extended to the case of an elongated body with an ellipsoidal shape. The semi-analytical model presented in this section is only an approximation of the complex phenomena that occur during a standoff explosion. A number of effects are not considered here and there are strong assumptions on the absorption of radiation and on the vaporisation process. In particular, we assume that the surface of the asteroid is composed of hard rock with low porosity. As in Solem (1993), we assume a linear relationship between the mass of the nuclear bomb and total yield, with all the energy absorbed by the material going into the vaporisation process, where only vaporisation is considered rather than melting and vaporisation. Furthermore, no shock wave propagation, reflection and diffraction are modelled. More accurate results can be found in the work of Plesko et al. (2010) who used a full numerical simulation. As with Shubin et al. (1995); Meshcheryakov (2014a), the model in this section is sufficient to get an estimation of the $\delta v$ 
imparted to the asteroid and serves the main scope of this paper, which is to compare a single detonation with a fractionated approach.

\subsection{Spherical Asteroid}

The energy released during the explosion is carried by the debris of the exploded spacecraft and by the radiations produced. Table 2.1 shows the fraction $f_{i}$ (with $i \in\{1,2,3,4,5\}$ ) of energy associated to each of the products of the explosion for the case of a fusion and fission devices (Glasstone, 1962; Hammerling and Remo, 1995).

Table 1: Energy fraction $f_{i}$ over all the products of a nuclear explosion

\begin{tabular}{lccccc}
\hline Source & 1-X-ray & 2-Neutrons & 3-Gamma rays & 4-Debris & 5-Others \\
\hline Fission & 0.7 & 0.01 & 0.02 & 0.2 & 0.07 \\
Fusion & 0.55 & 0.2 & 0.01 & 0.2 & 0.04 \\
\hline
\end{tabular}

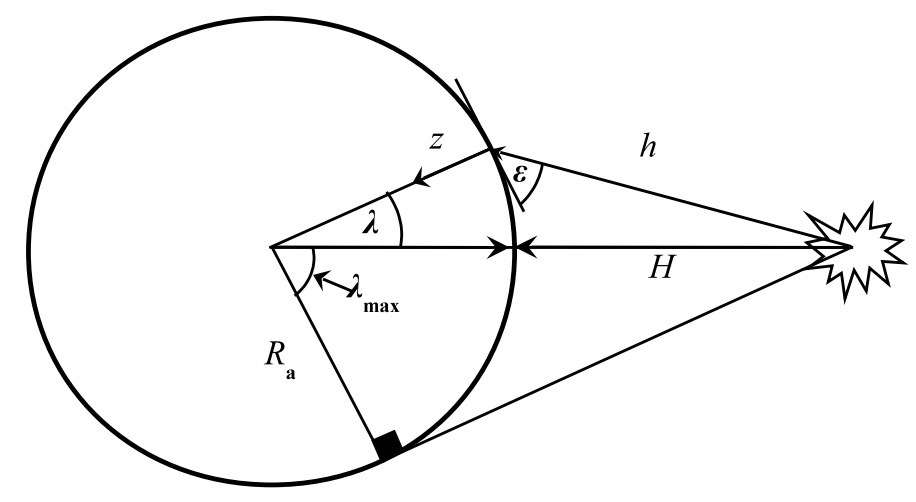

Figure 1: Standoff configuration for the nuclear interceptor method

The energy delivered during the explosion, $Y_{0}$, is computed from the yield-to-mass ratio and is conservatively assumed to have a value $Y T W=$ $0.75 \mathrm{ktons} / \mathrm{kg}$ for fusion devices and $Y T W=0.075 \mathrm{ktons} / \mathrm{kg}$ for fission devices $^{3}$.

$$
Y_{0}=Y T W m_{w h}
$$

\footnotetext{
${ }^{3}$ Based on data available online at nuclearweaponarchive.org.
} 
where $m_{w h}$ is the mass of the bomb. This assumption is more conservative than the one of Solem (1993). In this paper, no buried or surface detonation are considered due to the added difficulty of landing and digging on an asteroid.

With reference to Figure 1, the explosion is assumed to happen at a distance $H$ from the surface of the asteroid, therefore, only a portion $m_{\text {debris }}$ of the total mass of debris $m_{d}$ will hit the surface:

$$
m_{\text {debris }}=S m_{d}
$$

If one assumes that the exploding device sees a spherical cap with radius $R_{A}$, then the fraction $S$ can be expressed as:

$$
S=\frac{1}{2}-\frac{\sqrt{H}}{2} \frac{\sqrt{H+2 R_{A}}}{R_{A}+H}
$$

The ejection velocity of the debris $v_{\text {debris }}$ is then computed from the fraction $f_{4}=0.2$ (see Table 2.1) of the total energy $Y_{0}$ released during the blast:

$$
v_{\text {debris }}=\sqrt{\frac{2 f_{4} Y_{0}}{m_{d}}}
$$

The variation of velocity $\delta v_{\text {debris }}$ due solely to the debris cloud is then given by:

$$
\delta v_{\text {debris }}=\beta S_{s c} \frac{m_{\text {debris }} v_{\text {debris }}}{m_{A}}
$$

where $S_{s c}$ is a scattering factor and $\beta$ the momentum enhancement factor (Tedeschi, Remo, Schulze and Young, 1995) which was set to $\beta=2$ in the simulations.

The contribution from the radiation is derived from the Beer-Lambert law of absorption. Given a radiation with frequency $\nu$ and knowing the incident radiation energy per unit area $I_{0}^{\nu}(\lambda)$ and the depth $z$, the energy per unit area $I^{\nu}(\lambda, z)$ transmitted beyond a given depth is computed as follows:

$$
I^{\nu}(\lambda, z)=\sin \epsilon(\lambda) I_{0}^{\nu}(\lambda) \exp \left(-\rho_{A} \kappa_{\nu} \frac{z}{\sin \epsilon(\lambda)}\right)
$$

The incident radiation density $I_{0}^{\nu}(\lambda)$ is given by:

$$
I_{0}^{\nu}(\lambda)=\frac{f_{i}}{4 \pi h^{2}(\lambda)} Y_{0}
$$


where the $h$ distance is computed as:

$$
h=\sqrt{\left(H+(1-\cos \lambda) R_{A}\right)^{2}+R_{A}^{2} \sin ^{2} \lambda}
$$

and $\epsilon$ is given by:

$$
\sin \epsilon=\frac{\left(R_{A}+H\right) \cos \lambda-R_{A}}{h}
$$

The linear mass-absorption coefficient $\kappa_{\nu}$ for each type of radiation is given in Table 2 (Hammerling and Remo , 1995). Note that quantities in Table 2 are considered as mean values over the range of frequencies of X-rays and gamma-rays.

Table 2: Opacity $\kappa_{\nu}$, or linear mass-absorption coefficient, for an asteroid made of forsterite

\begin{tabular}{lccc}
\hline Radiation type & X-Ray & Neutron & Gamma ray \\
\hline Value & $1.5 \mathrm{~m}^{2} / \mathrm{kg}$ & $0.0044 \mathrm{~m}^{2} / \mathrm{kg}$ & $0.005 \mathrm{~m}^{2} / \mathrm{kg}$ \\
\hline
\end{tabular}

The amount of energy absorbed per unit mass at a given depth is then obtained by considering the cumulative absorption of each radiation type:

$$
E(\lambda, z)=-\sum_{\nu} \frac{d I^{\nu}}{d z}=\sum_{\nu} \kappa_{\nu} I_{0}^{\nu} \exp \left(-\rho_{A} \kappa_{\nu} \frac{z}{\sin \epsilon(\lambda)}\right)
$$

Part of this energy goes into the vaporization process of the asteroid, while the excess energy is converted into thermal excitation. The local average velocity of the gas molecules $\bar{v}$ is then estimated by an energy balance, where $E_{v}$ is the total vaporization enthalpy per unit mass:

$$
\bar{v}(\lambda, z)=\sqrt{2\left(E(\lambda, z)-E_{v}\right)}
$$

This allows one to define a limit depth $z_{\max }$ below which the vaporization process cannot continue as the energy absorbed is lower than the vaporization enthalpy. Given a certain distance $H$ and yield $Y_{0}$, the value of $z_{\max }$ is numerically computed by finding the value of $z$ that satisfies the relationship $E(\lambda, z)=E_{v}$ for each $\lambda$ considered.

The change in linear momentum generated by the expelled material is expressed, for an infinitesimal volume, as:

$$
d P=\frac{\cos \lambda}{2} \rho_{A} \bar{v}(\lambda, z) d V
$$


where the cosine function comes from the fact that we only retain the tangential component, and the $\frac{1}{2}$ factor is due to the assumption of an equiprobable scattering of the gas molecules from the ablated surface over a hemisphere. The area of a spherical cap is given by:

$$
S=2 \pi R_{A}^{2}(1-\cos \lambda)
$$

with the infinitesimal volume $d V$ given by:

$$
d V=2 \pi R_{A}^{2} \sin \lambda d z d \lambda
$$

Integrating Eq. (12) and dividing by the mass of the asteroid gives the change of velocity $\delta v_{\text {radiation }}$ due to radiation:

$$
\delta v_{\text {radiation }}=\frac{\pi R_{A}^{2}}{M_{A}} \int_{0}^{\lambda_{\max }} \int_{0}^{z_{\max }(\lambda)} \rho_{A} \bar{v}(\lambda, z) d z \sin \lambda \cos \lambda d \lambda
$$

Figure $2(\mathrm{a})$ shows the total $\delta v=\delta v_{\text {radiation }}+\delta v_{\text {debris }}$ imparted to an asteroid, with a mass and density from Table 3, assuming a fusion device of $600 \mathrm{~kg}$ at different altitudes of detonation and for different values of the enthalpy of vaporization. Figure 2(b), by comparison, shows the $\delta v$ imparted to the same asteroid by a fission device of equal mass, at different altitudes of detonation and for different values of the enthalpy of vaporization.

\subsection{Elongated Asteroid}

In this section, we consider the case of an elongated asteroid with an elongation factor $e_{l}$, defined as an ellipsoid with semi-major axis $a_{I}=e_{l}^{2 / 3} R_{A}$ and semi-minor axes $b_{I}=c_{I}=R_{A} e_{l}^{-1 / 3}$. The mean radius is identical to the one used in the spherical case previously derived, such that the elongated and spherical asteroids considered have identical volumes. Considering the configuration where the bomb is detonated along the longer side as a worst case scenario, the distance $h(\lambda)$ is now given as:

$$
h=\sqrt{\left(H+(1-\cos \lambda) e_{l}^{2 / 3} R_{A}\right)^{2}+\left(\frac{R_{A}}{e_{l}^{1 / 3}}\right)^{2} \sin ^{2} \lambda}
$$

We need now to distinguish between $\lambda$, the angle in elliptical coordinates corresponding to the concentric circle of radius $a_{I}$, and $\tilde{\lambda}$, the angle between 


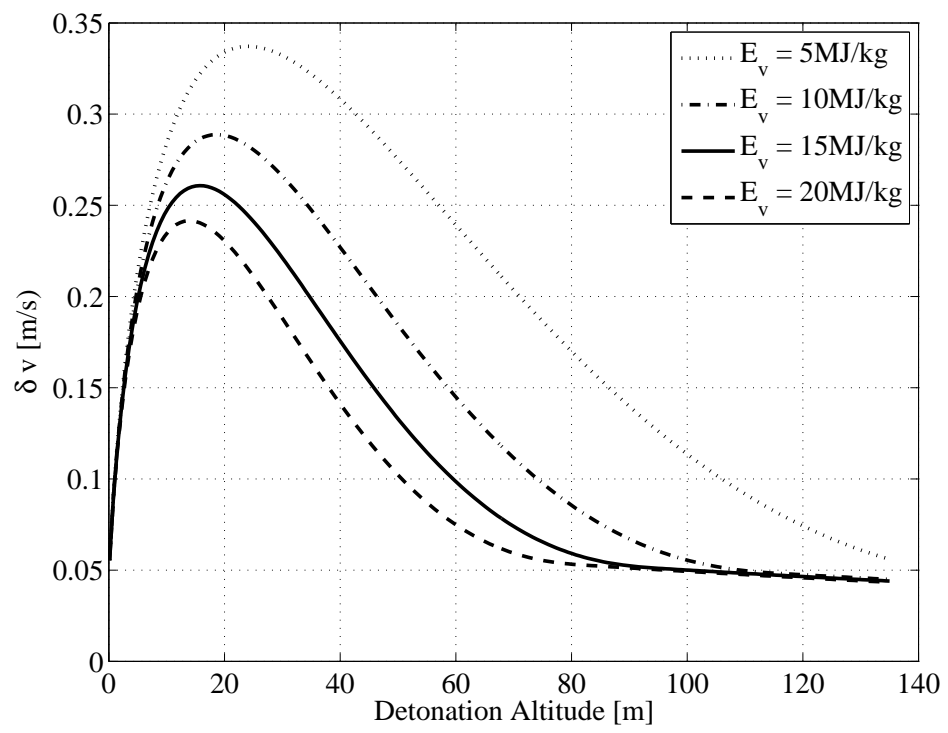

(a) $600 \mathrm{~kg}$ fusion device

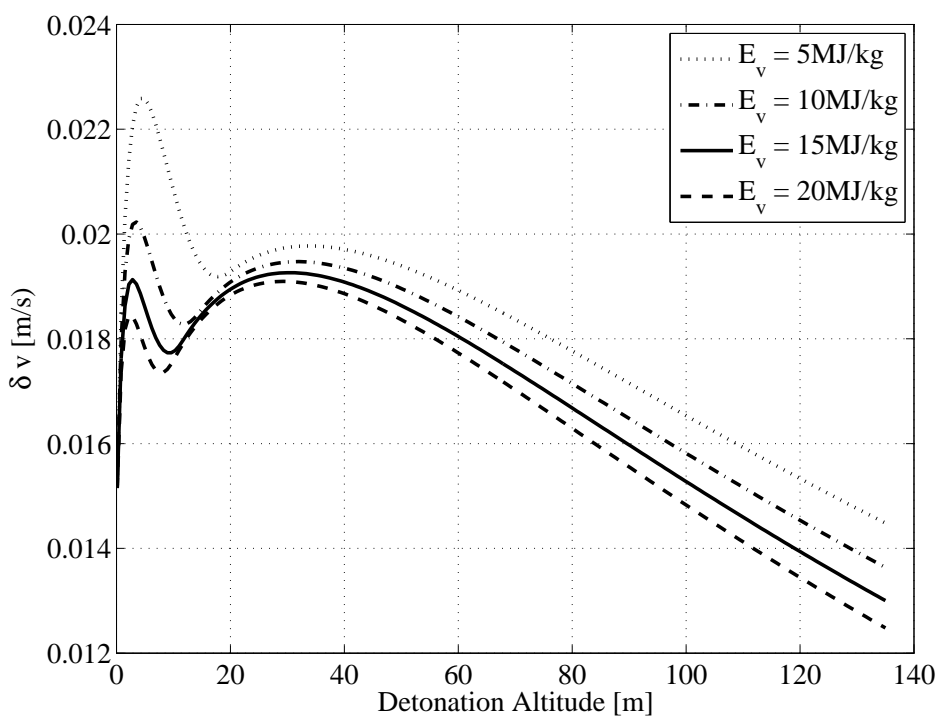

(b) $600 \mathrm{~kg}$ fission device

Figure 2: Impulsive change of velocity as a function of the detonation altitude for different values of the enthalpy of vaporization

the normal to the ellipsoidal surface and the horizontal direction. They can 
be related through the following formula:

$$
\cos \tilde{\lambda}=\frac{\cos \lambda}{\sqrt{1+\left(e_{l}^{2}-1\right) \sin ^{2} \lambda}}
$$

The value of $\sin \epsilon$ is obtained by computing the scalar product between the direction normal to the ellipsoidal surface $\mathbf{n}$ and the direction $-\mathbf{h}$, which gives

$$
\sin \epsilon=\frac{\frac{e_{l}^{2 / 3} R_{A}+H}{e_{l}^{2 / 3} R_{A}} \cos \lambda-1}{\frac{e_{l}^{1 / 3} h}{R_{A}} \sqrt{\frac{\cos ^{2} \lambda}{e_{l}^{2}}+\sin ^{2} \lambda}}
$$

The infinitesimal volume is now expressed as:

$$
d V=2 \pi e_{l}^{1 / 3} R_{A}^{2} \frac{\sin ^{2} \lambda}{\sin \tilde{\lambda}} d z d \lambda
$$

Keeping a constant detonation altitude of $17 \mathrm{~m}$, Figure 3 shows how the $\delta v$ produced compares to the spherical case, considering again a $600 \mathrm{~kg}$ fusion device.

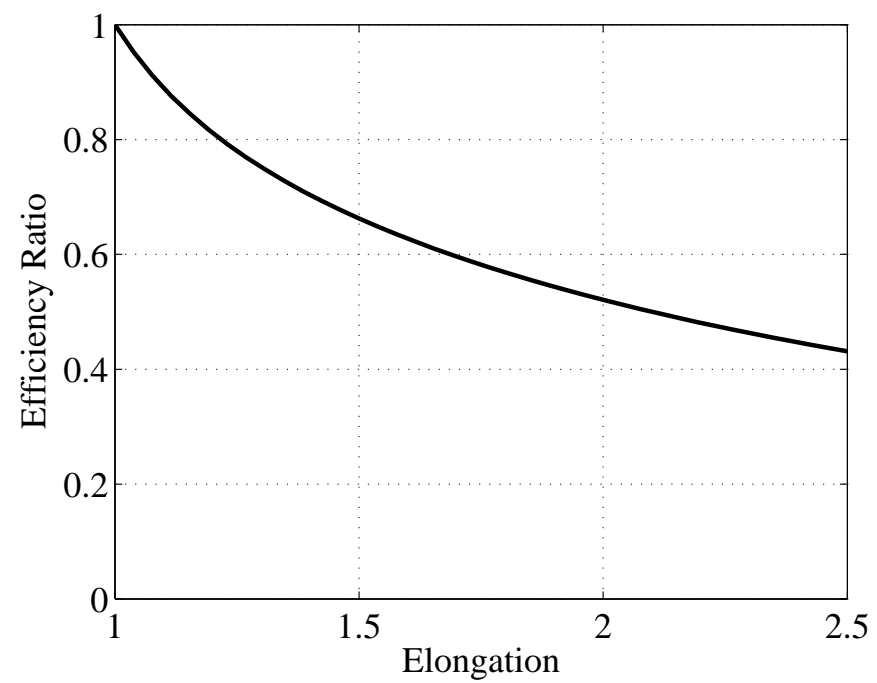

Figure 3: Performance comparison between the spherical case and a cigar-shaped asteroid as a function of the elongation 


\subsection{Comparison with Other Simplified Analytical Models}

As mentioned in the previous sections, the model proposed in this paper is largely based on the existing literature on the subject and represents only an approximation of the full phenomenon. It is however interesting to compare our predicted $\delta v$ against the prediction of two other simplified analytical formulations proposed by Solem (1993) and Meshcheryakov (2014a)Meshcheryakov (2014b).

The model in Solem (1993) is given by the simple parametric exponential law:

$$
\delta v=\frac{\alpha \delta}{m_{A}}\left(Y T W m_{w h}\right)^{\frac{\beta+1}{2}}
$$

where, using Solem's notation, the parameter $\alpha$ is the crater constant, $\beta$ the crater exponent and $\delta^{2} / 2$ is said to be the energy that goes into the dirt ejected from the crater. The values of the three parameters are derived experimentally. For the case of a stand-off explosion, Solem (1993) proposes the following values: $\beta=1, \alpha$ between $[1,2] \times 10^{-6} \mathrm{~s} / \mathrm{cm}$ and $\delta$ between 0.2 and 0.4. He also suggests that the most effective solution would be a neutron bomb with high neutron deposition, therefore we can compare the value computed with Eq. (20) against our model for neutron bombs. If we take the lower bounds, $\alpha=10^{-4} \mathrm{~s} / \mathrm{m}$ and $\delta=0.2$, then Eq. (20) gives $1.4 \mathrm{~m} / \mathrm{s}$ which is about 4 times higher than our prediction.

By comparison, Meshcheryakov's model gives an approximation of the $\delta v$ as:

$$
\delta v=Q^{0.554}\left(1-\frac{\sqrt{h^{2}+2 h R}}{R+h}\right) \frac{W}{m_{A}}
$$

where, using Meshcheryakov's notation, $Q$ is the yield of the explosion and $W$ is a tabulated factor that depends on the altitude of the explosion. Interpolating the values available in Meshcheryakov (2014a), at $25 \mathrm{~m}$ from an asteroid with radius of $134 \mathrm{~m}$ and assuming a fission device with a mass of $600 \mathrm{~kg}$, Eq. (21) predicts a $\delta v$ of $0.0189 \mathrm{~m} / \mathrm{s}$ which is consistent with the prediction of our model for a fission device with the same yield exploding at the same distance.

This simple comparison shows that the model proposed in this paper is sufficiently accurate and conservative to derive sensible conclusions on the utility of a fractionated approach. Furthermore, the use of multiple fractionated explosion would allow detonating the bombs deeper and deeper inside 
the asteroid, as part of the material is ejected at each explosion. Therefore, the effect of each explosion would progressively increase. This cratering effect, however, is not considered in the current model.

\section{Nuclear Cycler Mission Concept and Design}

The key idea is to incrementally change the velocity of the asteroid by releasing and detonating a series of relatively small nuclear bombs from a vantage point at a safe distance from the asteroid. Figure 3 shows a possible configuration with a carrier-spacecraft flying in formation with the asteroid on a periodic orbit at a set distance from the asteroid and releasing two bombs at two different times. The detonation occurs on the far side of the asteroid with respect to the spacecraft so that the asteroid is shielding the spacecraft from radiation and debris.

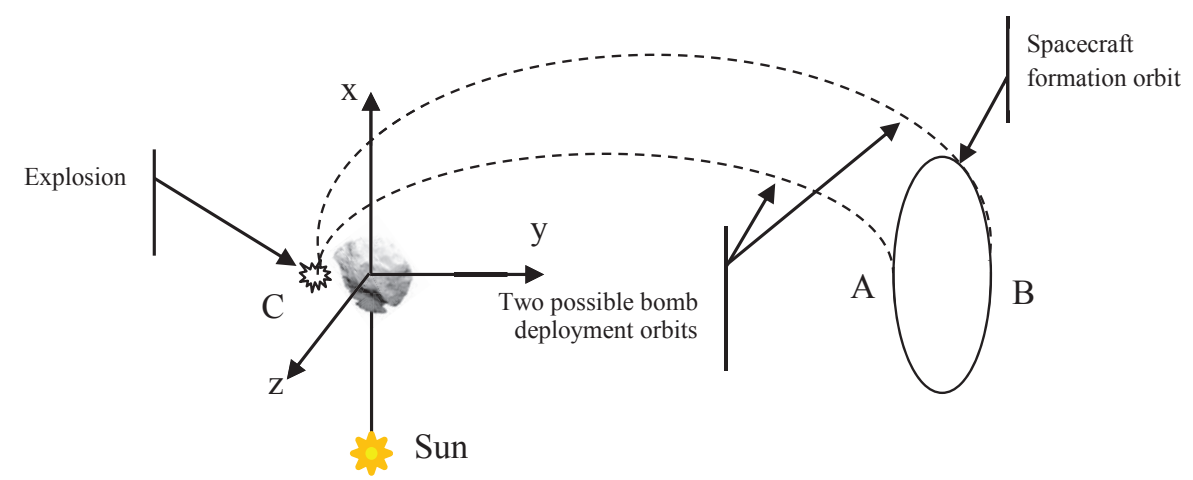

Figure 4: Illustration (not to scale) of the nuclear cycler concept

In this particular configuration, the orbit of the carrier and the one of the bomb are timed in such a way that by the time the bomb goes from point A to point $\mathrm{C}$, the carrier has moved from point A to point B. Equivalently, by the time the bomb goes from $\mathrm{B}$ to $\mathrm{C}$, the carrier has moved from $\mathrm{B}$ back to A, closing the cycle, after which a new cycle will begin. The data from the previous explosions can be collected and analysed to better control the altitude and timing of the subsequent explosions or to control the direction of the resulting $\delta \mathbf{v}$.

In the remainder of this paper we will analyse only the special configuration in which point $\mathrm{A}$ corresponds to the perihelion of the orbit of the 
asteroid, and point B to the aphelion. In this case two bombs are released every revolution of the asteroid around the Sun.

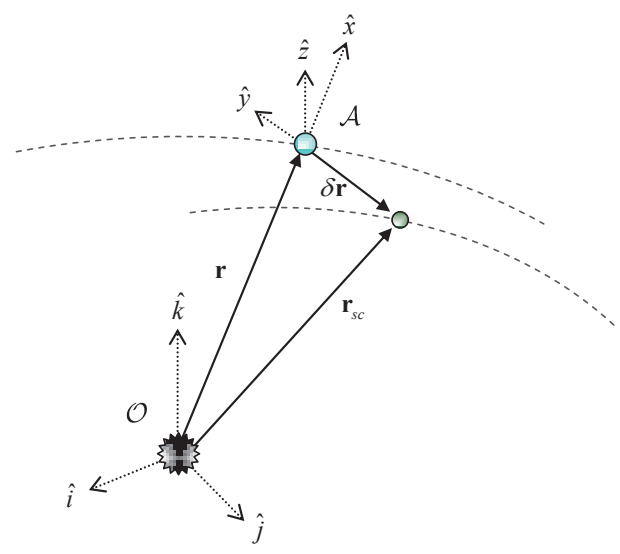

Figure 5: Local Hill's reference frame centred on the asteroid

\subsection{Preliminary Trajectory Design}

A first approximation of the trajectory of the spacecraft and bombs can be obtained using the linearised proximity motion equations (Schaub and Junkins , 2003):

$x \approx \frac{r}{a} \delta a+\frac{a e \sin \theta}{\sqrt{1-e^{2}}} \delta M-a \cos \theta \delta e$

$y \approx \frac{r}{\left(1-e^{2}\right)^{3 / 2}}(1+e \cos \theta)^{2} \delta M+r \delta \omega+\frac{r \sin \theta}{\left(1-e^{2}\right)}(2+e \cos \theta) \delta e+r \cos i \delta \Omega$

$z \approx r\left(\sin \theta^{*} \delta i-\cos \theta^{*} \sin i \delta \Omega\right)$

where $[a, e, i, \Omega, \omega]$ are the standard Keplerian orbit parameters of semimajor axis, eccentricity, inclination, right ascension (of the ascending node), and the argument of periapsis respectively, $\theta$ and $M$ are the true and mean anomalies, $\theta^{*}=\theta+\omega$ and $\delta \mathbf{r}=[x, y, z]^{T}$. The coordinate frames are shown in Figure 5 where $\mathcal{O}$ is a heliocentric inertial reference frame, and $\mathcal{A}$ is the relative Hill reference frame, centred on the asteroid. The vector $\delta \mathbf{k}=[\delta a, \delta e, \delta i, \delta \Omega, \delta \omega, \delta M]$ represents the difference in Keplerian elements between the orbits of the asteroid and spacecraft. 
In order to maintain the formation, the spacecraft and asteroid are put in a similar orbit around the Sun with identical semi-major axis so that $\delta a=0$, causing their orbital period to be equal and therefore there is no relative drift. The value of the remaining orbital parameters of the orbit of the carrier spacecraft can be computed by solving the following system of equations:

$$
\begin{array}{ll}
x(\theta=0)=0 & x(\theta=\pi)=0 \\
y(\theta=0)=y_{A} & y(\theta=\pi)=y_{B} \\
z(\theta=0)=0 & z(\theta=\pi)=0
\end{array}
$$

Given the proximity equations (22), one can see that system (23) can be satisfied if $\delta e=0, \delta i=0$ and $\delta \Omega=0$. In this case the problem reduces to the solution, for $\delta M$ and $\delta \omega$, of the following system of two equations:

$$
\begin{aligned}
& y(\theta=0)=\frac{r(\theta=0)}{\left(1-e^{2}\right)^{3 / 2}}(1+e)^{2} \delta M+r(\theta=0) \delta \omega=y_{A} \\
& y(\theta=\pi)=\frac{r(\theta=\pi)}{\left(1-e^{2}\right)^{3 / 2}}(1-e)^{2} \delta M+r(\theta=\pi) \delta \omega=y_{B}
\end{aligned}
$$

A first approximation of the trajectory of each of the bombs can be derived in a similar fashion assuming, this time, that:

$$
\begin{aligned}
& y(\theta=0)=\frac{r(\theta=0)}{\left(1-e^{2}\right)^{3 / 2}}(1+e)^{2} \delta M+r(\theta=0) \delta \omega=y_{A} \\
& y(\theta=\pi)=\frac{r(\theta=\pi)}{\left(1-e^{2}\right)^{3 / 2}}(1-e)^{2} \delta M+r(\theta=\pi) \delta \omega=y_{C}
\end{aligned}
$$

and

$$
\begin{aligned}
& y(\theta=\pi)=\frac{r(\theta=\pi)}{\left(1-e^{2}\right)^{3 / 2}}(1-e)^{2} \delta M+r(\theta=\pi) \delta \omega=y_{B} \\
& y(\theta=0)=\frac{r(\theta=0)}{\left(1-e^{2}\right)^{3 / 2}}(1+e)^{2} \delta M+r(\theta=0) \delta \omega=y_{C}
\end{aligned}
$$

Figure 6 shows an example of trajectories for the bombs and the spacecraft after setting $y_{B}=131 \mathrm{~km}, y_{A}=214 \mathrm{~km}$ and $y_{C}=-0.210 \mathrm{~km}$ respectively.

\subsection{Refined Trajectory Design}

The trajectories of the bombs in Figure 6 do not include the effects of the gravity of the asteroid. A corrected set of trajectories including the effect of gravity was then derived with a shooting approach by back-propagating the trajectory of the bomb from the desired detonation point to the carrier's orbit. As a first approximation, one can consider that the motion of the bombs remains planar and that the asteroid spins around an axis perpendicular to 


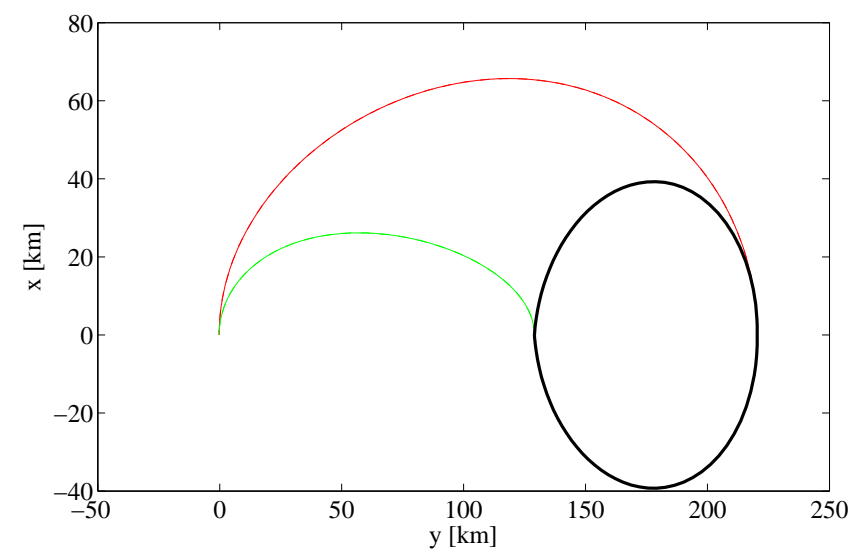

Figure 6: Example of trajectories of the bombs and the spacecraft in the Hill's reference frame with no perturbations from the gravity field of the asteroid

the orbit plane. In this case, only the in-plane components of the proximal motion dynamics are required:

$$
\begin{aligned}
& \ddot{x}=-\ddot{r}+2 \dot{\theta} \dot{y}+\dot{\theta}^{2}+\ddot{\theta} y-\frac{\mu_{\text {sun }}(r+x)}{r_{s c}^{3}}-\frac{\mu_{A}}{\delta r^{3}} x+\frac{\partial U_{20+22}}{\partial x} \\
& \ddot{y}=-2 \dot{\theta}(\dot{r}+\dot{x})-\ddot{\theta}(r+x)+\dot{\theta}^{2} y-\frac{\mu_{s u n}}{r_{s c}^{3}} y-\frac{\mu_{A}}{\delta r^{3}} y+\frac{\partial U_{20+22}}{\partial y} \\
& \ddot{z}=-\frac{\mu_{s u n}}{r_{s c}^{3}} z-\frac{\mu_{A}}{\delta r^{3}} z+\frac{\partial U_{20+22}}{\partial z}
\end{aligned}
$$

with

$$
\begin{aligned}
& \ddot{\theta}=-\frac{2 \dot{r} r \dot{\theta}}{r^{2}} \\
& \ddot{r}=\dot{\theta}^{2} r-\frac{\mu_{\text {sun }}}{r^{2}}
\end{aligned}
$$

where $\mu_{\text {sun }}$ and $\mu_{A}$ are the gravity constants of the Sun and the asteroid, respectively. The asteroid shape was assumed to be an ellipsoid with $a_{I} \geq$ $b_{I}=c_{I}$, the three semi-axes along the three body-fixed orthogonal axes, as in Section 2.2. The value of the semi-axes is given in Table 3. Note that the total expelled mass is negligible compared to the mass of the asteroid, thus we used the conservative assumption that the mass of the asteroid remains unchanged. Furthermore, in the reduced model presented in this paper, the spinning rate and axis are assumed to remain constant.

The axis $c_{I}$ is assumed to be aligned with the vector of angular momentum, which corresponds to the $z$-axis of the asteroid Hill frame $\mathcal{A}$ (see Figure 5). The gravity field of the asteroid is expressed as the sum of a spherical field $\mu_{A}(\delta r)^{-2}$ plus a second-degree and second-order field (Hu and Scheeres , 
Table 3: Orbital and physical properties of test asteroid

\begin{tabular}{lll}
\hline \multicolumn{1}{c}{ Element } & & Measured Value \\
\hline Semi-major axis & $a_{0}$ & $0.9224 \mathrm{AU}$ \\
Eccentricity & $e_{0}$ & 0.1912 \\
Period & $T_{0}$ & $323.5969 \mathrm{days}$ \\
Mean motion & $n_{0}$ & $1.2876 \times 10^{-5} \mathrm{deg} / \mathrm{s}$ \\
\hline Mass & $m_{A}$ & $2.7 \times 10^{10} \mathrm{~kg}$ \\
Gravitational constant & $\mu_{A}$ & $1.801599 \times 10^{-9} \mathrm{~km}^{3} / \mathrm{s}^{2}$ \\
Physical dimensions & $a_{I}, b_{I}, c_{I}$ & $196 \mathrm{~m}, 112 \mathrm{~m}, 112 \mathrm{~m}$ \\
Rotational velocity & $w_{A}$ & $3.3 \times 10^{-3} \mathrm{deg} / \mathrm{s}$ \\
Total vaporisation Enthalpy & $E_{v}$ & $15 \mathrm{MJ} / \mathrm{kg}$ \\
Density & $\rho_{A}$ & $2650 \mathrm{~kg} / \mathrm{m}^{3}$ \\
\hline
\end{tabular}

2002; Rossi et al. , 1999):

$$
U_{20+22}=\frac{\mu_{A}}{\delta r^{3}}\left[C_{20}\left(1-\frac{3}{2} \cos ^{2} \varphi\right)+3 C_{22} \cos ^{2} \varphi \cos 2 \alpha\right]
$$

where $\varphi$ is the elevation over the $x-y$ plane and the harmonic coefficients $C_{20}$ and $C_{22}$ are a function of the semi-axes,

$$
\begin{aligned}
& C_{20}=-\frac{1}{10}\left(2 c_{I}^{2}-a_{I}^{2}-b_{I}^{2}\right) \\
& C_{22}=\frac{1}{20}\left(a_{I}^{2}-b_{I}^{2}\right)
\end{aligned}
$$

and $\alpha$ is defined as,

$$
\alpha=\arctan \left(\frac{y}{x}\right)+w_{A} t
$$

The initial conditions for the backward integration, in the case when the bomb is released from point $\mathrm{B}$, are:

$$
\begin{aligned}
& x(\theta=0)=0 \\
& y(\theta=0)=y_{C} \\
& z(\theta=0)=0 \\
& \dot{x}(\theta=0)=\delta v_{C} \cos \gamma \\
& \dot{y}(\theta=0)=\delta v_{C} \sin \gamma \\
& \dot{z}(\theta=0)=0
\end{aligned}
$$

The modulus of the velocity $\delta v_{C}$ was varied from $\left(v_{e x}+\epsilon_{e x 1}\right)$ to $\left(v_{e x}+\epsilon_{e x 2}\right)$, with $v_{e x}=\sqrt{2 \mu_{A} / y_{c}}$ at distance $y_{C}$ from the centre of the asteroid, while the 
$\gamma$ angle was constrained to be in the interval $[0.6, \pi / 2] \mathrm{rad}$. The trajectory was then propagated backward for a true anomaly $\Delta \theta=\pi$ using an adaptive Runge-Kutta, Dormand-Prince scheme implemented in the Matlab function ode 45 , with absolute and relative tolerance equal to $1 \mathrm{e}-6$ and $1 \mathrm{e}-7$ respectively. If the bomb is released from point $\mathrm{B}$, the arrival conditions need to satisfy the constraints:

$$
x(\theta=\pi)^{2}+\left(y(\theta=\pi)-y_{B}\right)^{2}+z(\theta=\pi)^{2}=0
$$

This analysis, however, assumes that for each value of $\delta v_{C}$, a particular spacecraft formation orbit is targeted, rather than targeting always the same orbit. Therefore, the Matlab function fmincon was used to find the optimal value of $\gamma$ that satisfies the relaxed soft constraint $x(\theta=\pi)^{2}+z(\theta=\pi)^{2}=0$ for different values of $\delta v_{C}$. As it is shown in Figure 7 , this yields a continuous and compact set of trajectories. The minimum distance from the asteroid however is limited by the need to have the bomb completing the transfer in half a revolution of the asteroid around the Sun. For low values of $\delta v_{C}$, the gravity of the asteroid accelerates the velocity of the bomb leading to a crossing of the $y$-axes in less than half a period. The value of $\delta v_{C}$ in this analysis, therefore, was set to quickly clear the Hill's sphere of influence of the asteroid.

Figure 7 shows a set of optimised trajectories superimposed to the theoretical ones obtained using the linear proximity equations. Figure 7 (a) shows the case in which $y_{C}=-0.1287 \mathrm{~km}, \epsilon_{e x 1}=-1.44917 \times 10^{-5} \mathrm{~km} / \mathrm{s}$ and $\epsilon_{e x 2}=-1.42417 \times 10^{-5} \mathrm{~km} / \mathrm{s}$ while Figure $7(\mathrm{~b})$ shows the case in which $y_{C}=-0.210 \mathrm{~km}, \epsilon_{e x 1}=8.1178 \times 10^{-6} \mathrm{~km} / \mathrm{s}$ and $\epsilon_{e x 2}=8.3678 \times 10^{-6} \mathrm{~km} / \mathrm{s}$. These two cases correspond to an optimal explosion along the semi-minor and semi-major axes of the asteroid respectively. Note that, according to Figure 2, a variation of $81.3 \mathrm{~m}$ in the detonation altitude significantly decreases the effectiveness of the explosion. On the other hand, it is possible to find trajectories from point $\mathrm{A}$ or point $\mathrm{B}$ to any point $\mathrm{C}$ in the interval $[-0.1287,-0.210] \mathrm{km}$ hence it is possible to target the optimal detonation distance for each explosion.

Figure 8 shows a close-up of Figure $7(\mathrm{a})$ around point C. One can see that the bomb would approach the asteroid almost head on, although $\delta v_{C}$ is so small that was neglected in the calculation of the deflection $\delta v$. In this, analysis the assumption is that the bomb has a guidance, navigation and control system that can compensate for any additional effect, like light pressure. 


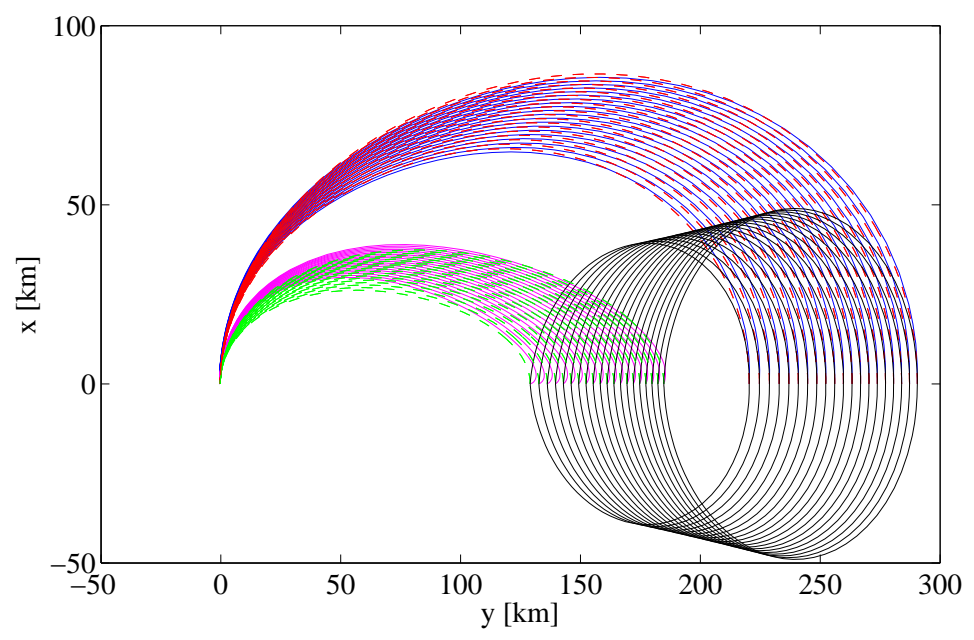

(a)

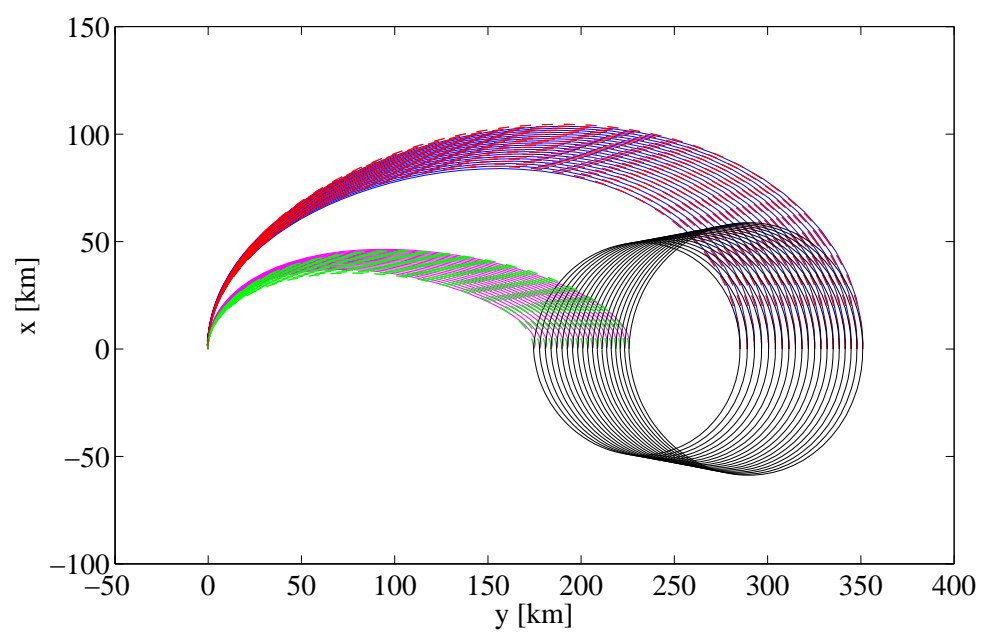

(b)

Figure 7: Families of formation and deployment trajectories for spacecraft and bombs: (a) detonation point at $210 \mathrm{~m}$, (b) detonation point at $128 \mathrm{~m}$. Magenta and blue curves are the true trajectories corrected for the gravity of the asteroid, the trajectory of the spacecraft is in black, and the blue, red and green curves show the estimated trajectories from the linear model.

The propellent cost to deploy each bomb can be evaluated by computing the velocity difference at points $\mathrm{A}$ and $\mathrm{B}$ of the trajectory of the bomb 


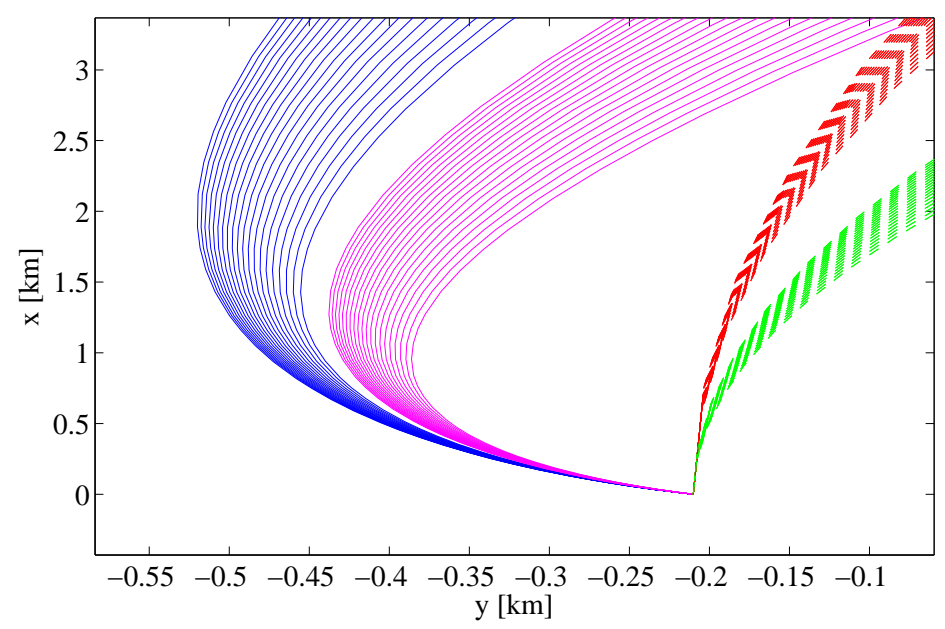

Figure 8: Close up of the families of deployment trajectories for a detonation point at $210 \mathrm{~m}$. Magenta and blue curves are the true trajectories corrected for the gravity of the asteroid, the blue, red and green curves are the estimated trajectories from the linear model.

and the trajectory of the spacecraft. The magnitude of the deployment $\Delta v$ manoeuvre, at the beginning of the deflection cycle, is reported in Figure 9 for point $\mathrm{A}$ and $\mathrm{B}$ and for the two detonation altitudes. This translates into a maximum mass fraction of $5.546 \times 10^{-5}$ per bomb assuming a cold gas engine with an $I_{s p}=68 \mathrm{~s}$. Finally, the formation orbit of the carrier designed with the linear proximity motion equations (22) were re-optimised to keep into account the full dynamics.

A small $\Delta \mathbf{v}$ correction manoeuvre was inserted at A and B to match the A-to-B trajectory with the B-to-A trajectory such that the A and B point remain the initial conditions of the bomb deployment trajectories and the periodicity of the formation orbit is maintained. The optimised correction manoeuvres, however, are of negligible size, even lower than the deployment manoeuvres, and are not reported here.

\subsection{Estimation of the Miss Distance}

Given the variation of the velocity of the asteroid $\delta \mathbf{v}=\left[\delta v_{t}, \delta v_{n}, \delta v_{h}\right]^{T}$ expressed in the tangential, normal and perpendicular to the orbit plane directions, at time $t_{d}$, one can compute the total variation of the orbital 


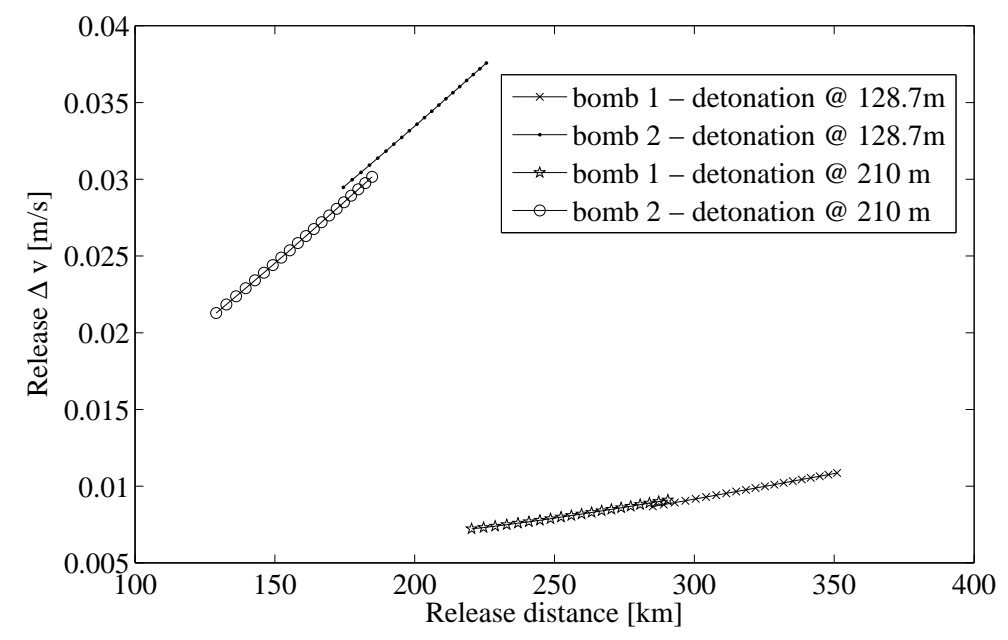

Figure 9: Total $\Delta v$ at the point of release of the bomb from the spacecraft

elements at a time $t_{\text {end }}$ (Vasile and Colombo , 2008):

$$
\begin{aligned}
\delta a & =\frac{2 a^{2} v}{\mu_{\text {sun }}} \delta v_{t} \\
\delta e & =\frac{1}{v}\left[2(e+\cos \theta) \delta v_{t}-\frac{r}{a} \sin \theta \delta v_{n}\right] \\
\delta i & =\frac{r \cos \theta}{\sqrt{\mu_{\text {sun }} p}} \delta v_{h} \\
\delta \Omega & =\frac{r \sin \theta}{\sqrt{\mu_{\text {sun }} p \sin i} \delta v_{h}} \\
\delta \omega & =\frac{1}{v e}\left[2 \sin \theta \delta v_{t}+\left(2 e+\frac{r}{a} \cos \theta\right) \delta v_{n}\right]-\frac{r \sin \theta^{*} \cos i}{\sqrt{\mu_{\text {sun }} p} \sin i} \delta v_{h} \\
\delta M & =\frac{\overbrace{\sqrt{\left(1-e^{2}\right)}}^{v e}\left[2\left(1+\frac{e^{2} r}{p}\right) \sin \theta \delta v_{t}+\frac{r}{a} \cos \theta \delta v_{n}\right]}{\text { geometric variation }}+\underbrace{\delta n\left(t_{\text {end }}-t_{d}\right)}_{\text {variation in a }}
\end{aligned}
$$

where

$$
\delta n=\sqrt{\frac{\mu_{\text {sun }}}{a^{3}}}-\sqrt{\frac{\mu_{\text {sun }}}{(a+\delta a)^{3}}}
$$

and $p=a\left(1-e^{2}\right)$. The time $t_{\text {end }}$ corresponds to the time between the next detonation and the last detonation, after which $t_{\text {end }}=t_{M O I D}$, i.e., the 
final time is the expected time of the impact of the un-deviated asteroid with the Earth. At each new detonation, therefore, all the orbital elements of the asteroid are updated with the variation computed with Eqs. (32). Then, given the cumulative variation of the orbital elements at $t_{M O I D}$, one can compute the displacement $\delta \mathbf{r}=\left[\begin{array}{lll}x & y & z\end{array}\right]^{T}$ of the asteroid in the Hill's reference frame centred in the un-deviated asteroid at the true anomaly $\theta$ of the MOID using Eqs. (22).

From the deflection $\delta \mathbf{r}$ the impact parameter $b$ at the time of the MOID can be computed(see Figure 10(a) where $\mathbf{V}_{N E O}$ is the velocity of the deviated asteroid with respect to the Earth). The impact plane can be defined as the plane centred in the Earth and perpendicular to the velocity vector of the un-deviated asteroid with respect to the Earth, $\mathbf{U}_{N E O}$, at the time of the impact (see Figure 10(b) where $\mathbf{v}_{E}$ is the velocity of the Earth). The deflection vector $\mathbf{x}_{b}$ in the $b$-plane coordinates can be expressed as:

$$
\mathbf{x}_{b}=\left[\begin{array}{lll}
\xi & \eta & \zeta
\end{array}\right]^{T}=\left[\begin{array}{lll}
\widehat{\xi} & \widehat{\eta} & \widehat{\zeta}
\end{array}\right]^{T} \delta \mathbf{r}
$$

where

$$
\widehat{\eta}=\frac{\mathbf{U}_{N E O}}{U_{N E O}}, \quad \hat{\xi}=\frac{\mathbf{v}_{E} \wedge \widehat{\eta}}{\left\|\mathbf{v}_{E} \wedge \widehat{\eta}\right\|}, \quad \hat{\zeta}=\widehat{\xi} \wedge \widehat{\eta}
$$

The impact parameter $b$ is then defined as:

$$
b=\sqrt{\xi^{2}+\zeta^{2}}
$$

At every explosion, the velocity of the asteroid is modified along with its orbital elements. As a consequence, the carrier needs to manoeuvre to maintain its relative orbital motion with respect to the asteroid. For the strategy presented in this paper, there is no out-of-plane component of the deflection, therefore, the spacecraft needs only an in-plane $\Delta \mathbf{v}_{c}$ correction. The component of this correction along the tangential direction has to compensate for the variation of the semi-major axis of the asteroid and, therefore, is of the same magnitude of $\delta v_{t}$ in Eq. (32). Given that the explosion occurs at the apsidal points, then $\delta v_{t}=\delta v$. Therefore, it is assumed that the spacecraft compensates only for a variation in $a$ and $e$ with a single manoeuvre equal to $\delta v$ at each explosion. This does not restore completely the exact initial proximal motion but provide sufficient control to maintain the formation with the asteroid. The result in Figure 7 demonstrates that one can generate a continuous set of formation orbits, at different distances from the asteroid, 


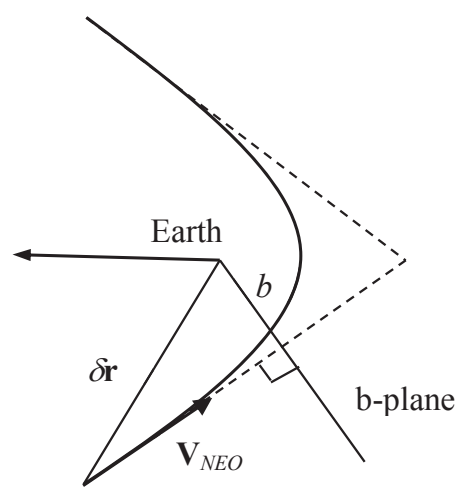

(a)

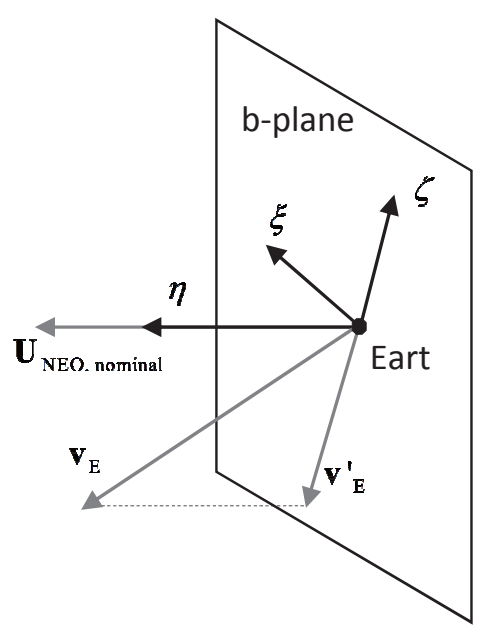

(b)

Figure 10: The impact plane and $b$ parameter

reaching the detonation point with different values of $\delta v_{C}$, hence at every explosion a new bomb deployment trajectory can be defined provided that the spacecraft maintains formation.

\section{Comparison Between a Single Detonator and the Nuclear Cycler}

The nuclear cycler method has been applied to the case of an Apophislike asteroid considering two different warning times of 6 years and 3 years respectively. The warning time is defined as the time from the first explosion to the expected impact of the un-deviated asteroid with the Earth. Relevant properties of this asteroid can be found in Table 3. The initial inclination $i$, right ascension of the ascending node $\Omega$, argument of the pericentre $\omega$ and mean anomaly $M$ were set so that the asteroid impacts the Earth on 13140 MJD2000.

An interesting initial result is obtained by computing the total $\delta v$ produced by either a single or a fractionated detonation for the same total mass of the bombs. The results of our model, in Figure 11, indicates that a fractionated explosion is better than a single explosion for the same total mass. The explanation of this result lies in the dependency of the $\delta v$ on the view angle $\lambda$ in Eq. (15), and the penetration depth $z_{\max }$. Figure 12 shows the optimal detonation altitude as a function of the spacecraft mass for a sin- 


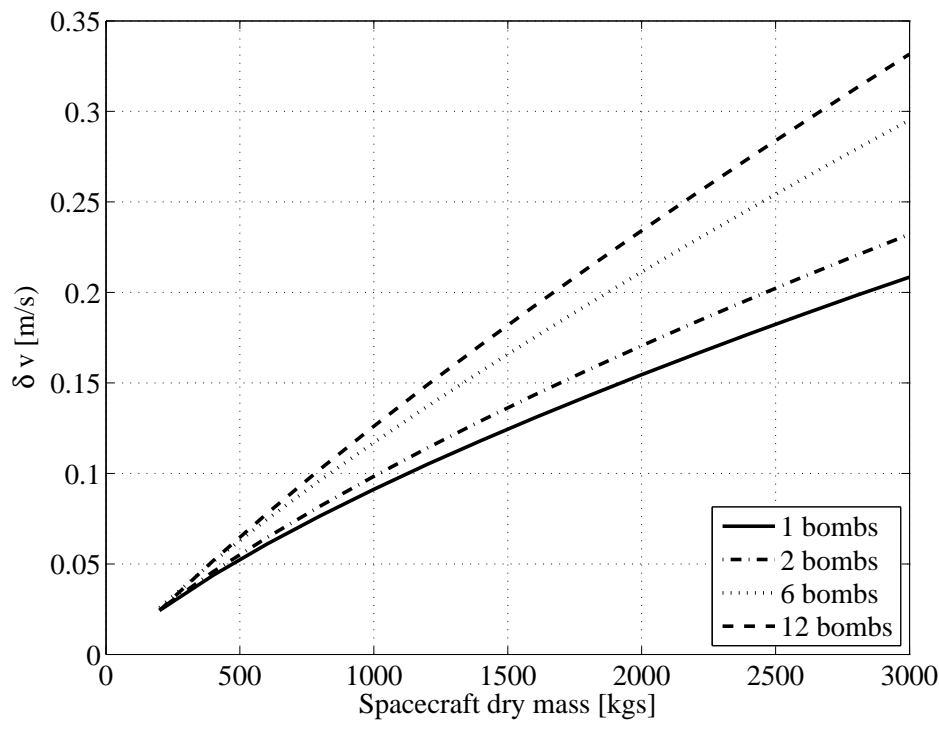

Figure 11: Total $\delta v$ as a function of the dry mass of the spacecraft for different numbers of explosions

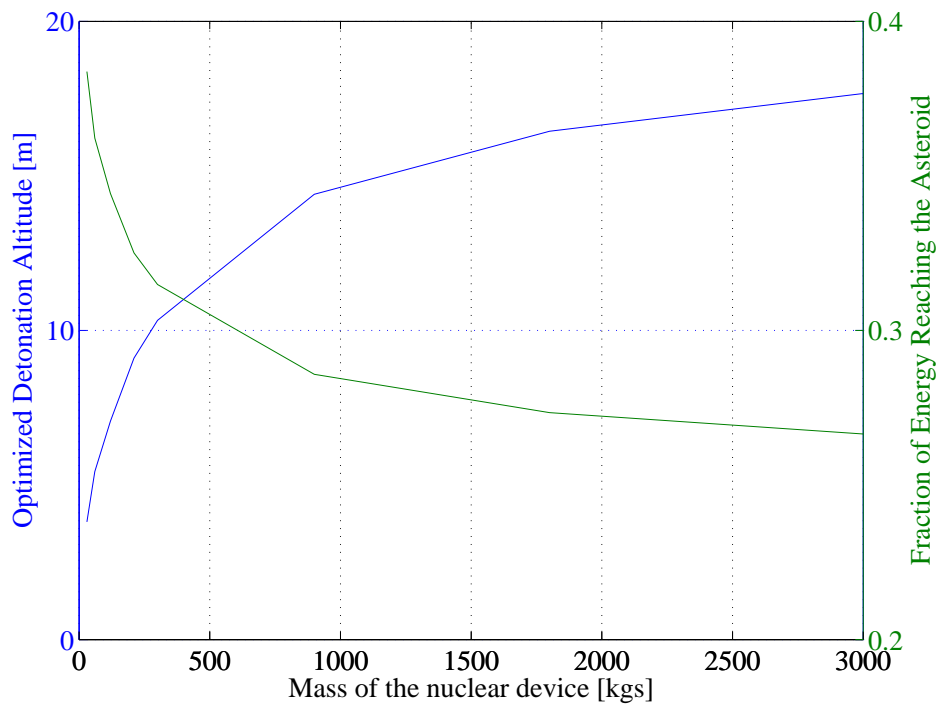

Figure 12: Optimal altitude of detonation and fraction of the total energy reaching the asteroid for different sizes of the nuclear device

gle interceptor. One can see that the optimal altitude is indeed lower for a smaller bomb, therefore, the fraction of the total released energy actually 
reaching the asteroid is larger in this case.

Using instead the deflection parameter $b$ as a performance indicator, shown in Figures 13 and 15, the single interceptor method outperforms the cycler one thanks to the fact that the total velocity variation is delivered at the very beginning of the first cycle and thus its effect propagates for a longer period. The comparison is done considering identical dry masses of the spacecraft with the cumulative mass of the nuclear bombs representing $30 \%$ of the total dry mass of the spacecraft in both cases. For the single interceptor method, the total mass of the spacecraft contributes to the ejecta, whereas only the mass of the bombs contributes to ejecta for the cycler method. Last but not least, in both cases, the detonation occurs at the optimal altitude.

As one would expect, Figures 13 and 15 show that a longer warning time is beneficial in term of deflection distance. A warning time of only 3 years constrains the maximum number of explosion to 6 for the nuclear cycler method if explosions occur only at the apsidal points.

Another interesting result is obtained by normalising the value of the $b$ parameter obtained for the case of a fractionated detonation with the result of the single interceptor method. The results in Figures 14 and 16 indicate that the $b$-parameter ratio can be as low as $40 \%$ for small spacecraft and reduces to $75 \%$ for larger spacecraft. The curves for 2 and 6 explosions overtake each other for the 6 years lead time case when the mass is larger than 1 ton.

\section{Discussion on the Technological Limitations and Requirements of the Proposed Approach}

While discussing the results of the nuclear cycler approach to asteroid deflection, it is important to keep in mind the initial purpose of this method: to partially bridge the existing gap between impulsive methods and slowpush strategies. If one looks at the $b$-parameter ratio, the nuclear cycler was demonstrated to be less efficient than a single detonation, although for the cases investigated in this paper, our results indicate that the performance of the nuclear cycler can remain relatively close to that of the single interceptor. Two opposing effects contribute to the overall performance of the nuclear cycler: i) the optimal detonation altitude in a sequence of small explosions is lower than for a single bigger one, thus the fraction of energy released during each explosion that impinges on the asteroid surface is higher; ii) the effect of each explosion propagates for less time. This latter effect alone explains why, despite an augmented total $\delta v$, the performance of the cycler strategy 


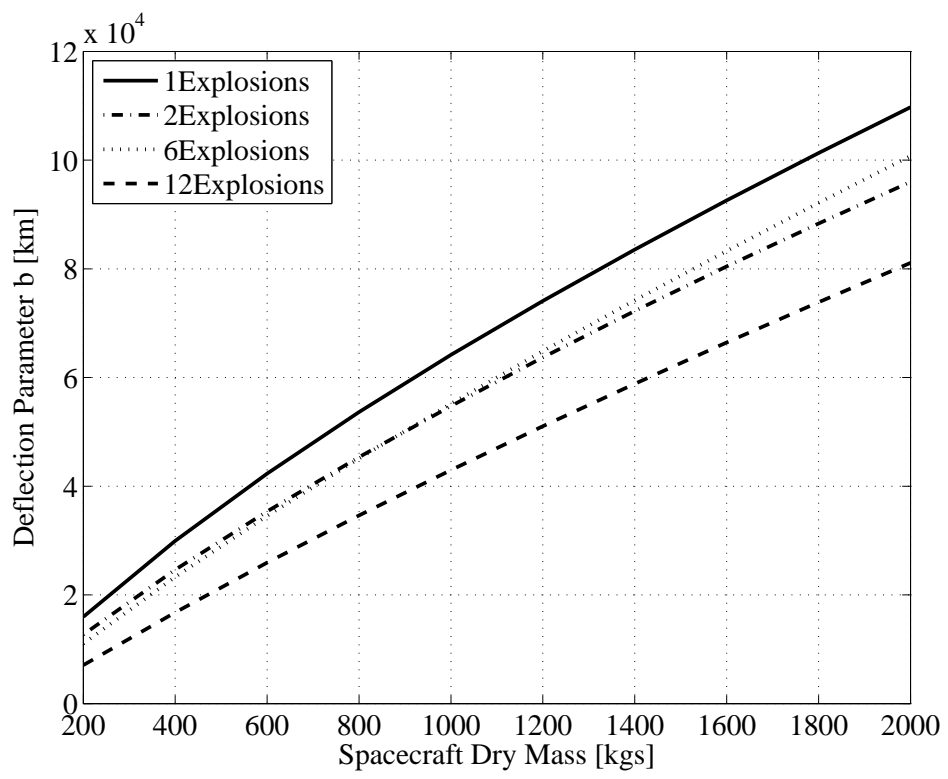

Figure 13: Deflection parameter for a varying number of explosions and a 6 years lead time

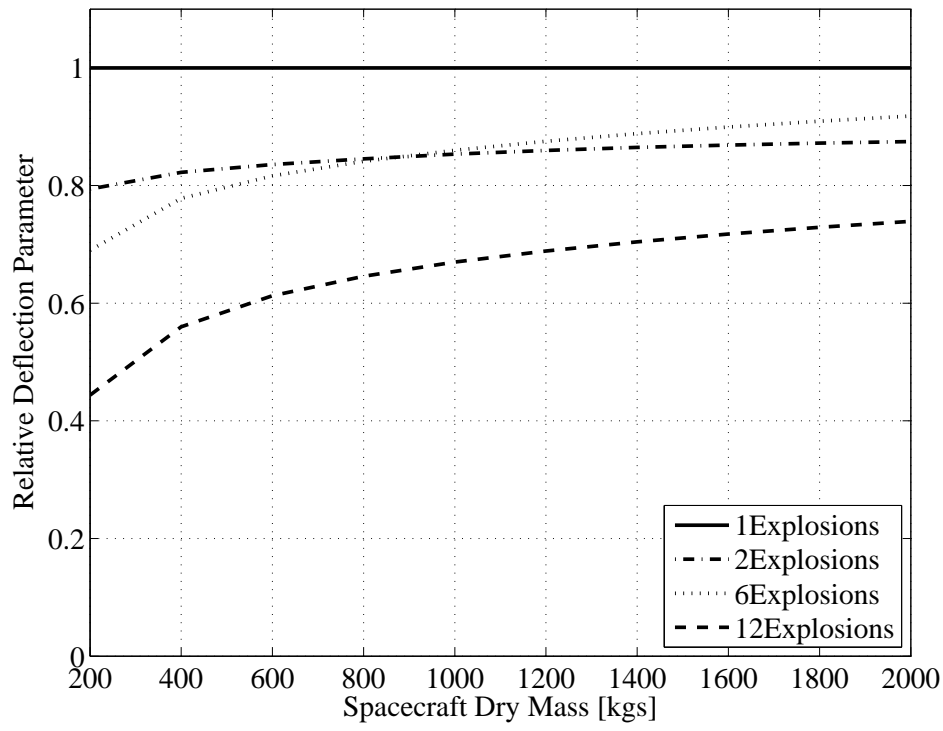

Figure 14: Efficiency of the nuclear cycler method compared to the single interceptor method for a 6 years lead time 


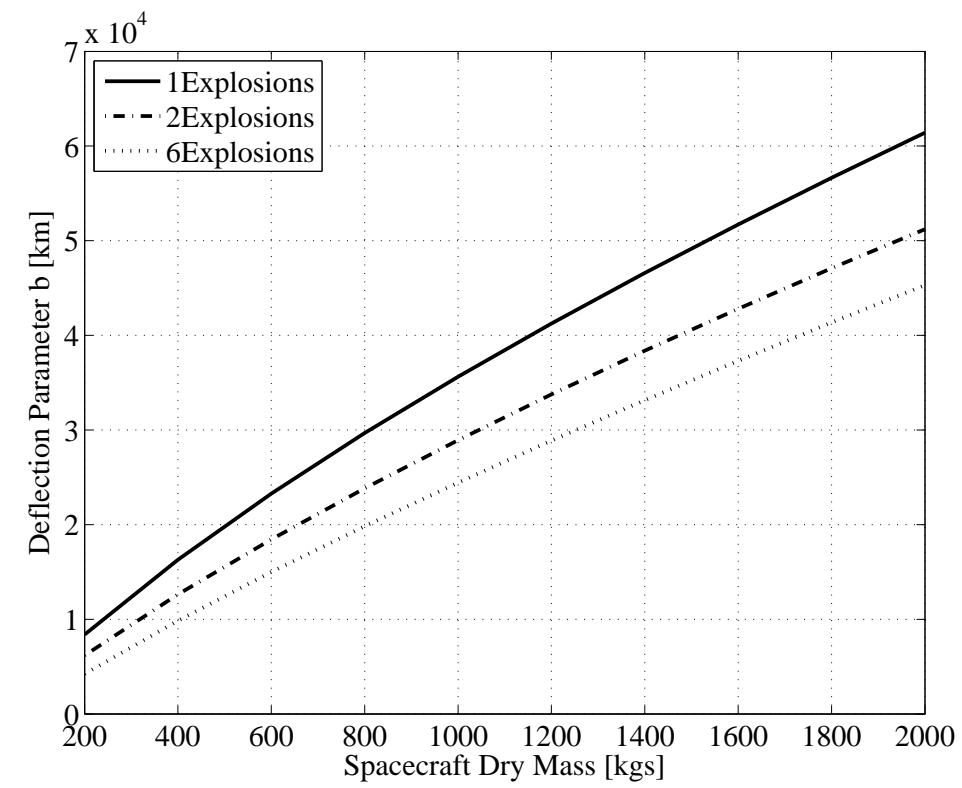

Figure 15: Deflection parameter for a varying number of explosions and a 3 years lead time

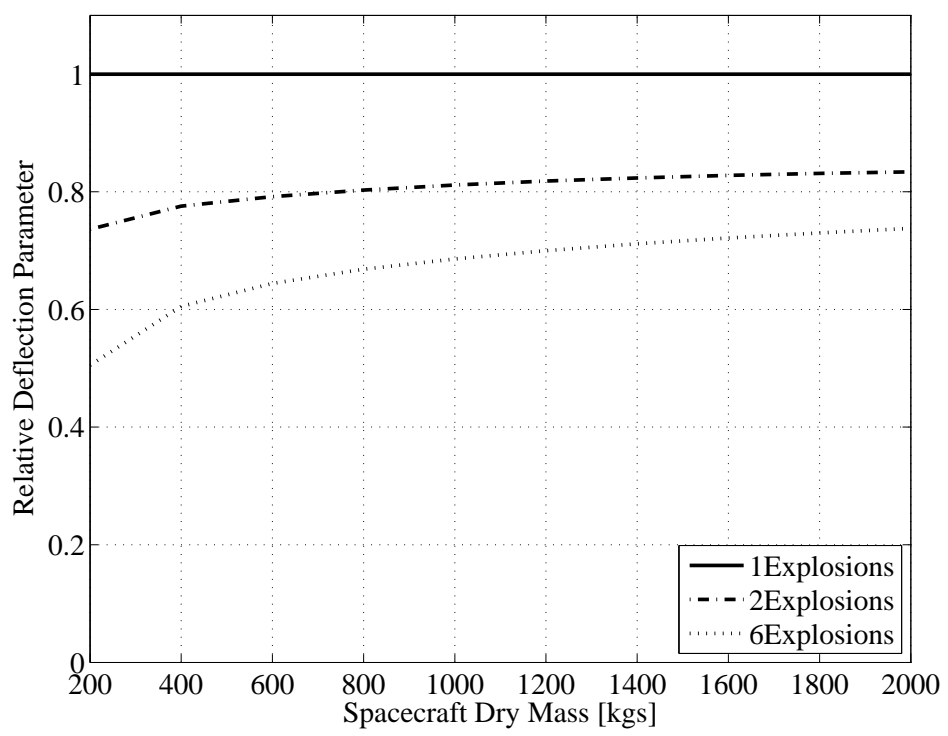

Figure 16: Efficiency of the nuclear cycler method compared to the single interceptor method for a 3 years lead time 
is not as good as the single impulsive method. The nuclear cycler does offer a higher degree of redundancy and controllability that, in our opinion, largely outweighs the performance loss, provided that sufficient warning time is available.

Compared to slow push methods, the nuclear cycler still maintains an edge due to the higher energy density. Although it requires similar navigation and control capabilities to maintain formation, it is not constrained to remain at a close distance from the asteroid, compared to other slow push methods, does not suffer from contamination effects and is less sensitive to the distance from the Sun.

A fractionated approach offers a further interesting advantage. If properly timed, each explosion excavates an increasingly deeper crater on the surface. As the altitude can be optimised for each bomb, each explosion can be designed to occur deeper into the core of the asteroid, transforming the initial stand-off explosion in a buried explosion. In this case, following the investigations of Meshcheryakov (2014a)Meshcheryakov (2014b) and Solem (1993), one can expect a progressive increase in performance.

In the following subsections we analyse three possible shortcomings of the proposed approach and the possible technological solution they require. In particular we consider the ejection of material from the side of the asteroid opposite to the explosion, the possible transient radiation effects on electronics, and the radiation effects on the spacecraft post explosion. In the last subsection, a failure analysis is conducted in which the whole nuclear cycler systems is suppose to completely fail after an explosion so that no further detonation is possible.

It is important to stress that each of the analyses in the following subsections does not represent an accurate and exhaustive description of the phenomenon but rather what can be considered as a limit case to be used only to identify possible remediation and requirements at system level. An exhaustive and accurate analysis of the phenomena analysed in this section require a separate investigation, which will be the subject of future studies.

\subsection{Lofting of Regolith}

The ejection of material due to the explosion is expected to generate a seismic wave. The seismic wave will travel through the asteroid and eventually lift a layer of regolith. The acceleration imparted to the regolith could be enough to reach the escape velocity and propel the regolith on the side opposite to the explosion, towards the spacecraft. 
A complete simulation of the propagation of the seismic wave generated by the explosion is out of the scope of this paper, however as a preliminary analysis, Martin et al. (2008) shows that for homogeneous asteroids the seismic wave, mainly the $p$-wave, propagates from the site of the explosion to the antipodal side and is then reflected back if the momentum is not transferred to the regolith. One can then take the limit case in which the propagation is uniform and the asteroid is spherical (a non-spherical asteroid would focus part of the seismic wave in preferential directions), the whole momentum is transferred to the regolith through the seismic wave and the asteroid velocity does not change. This assumption implies a seismic efficiency equal to 1 , such that the whole energy goes into $p$-waves and the seismic waves distribute over the whole antipodal hemisphere.

A further assumption is that the regolith forms a single layer of pebbles evenly distributed over the antipodal surface. This assumption would give a distribution of the regolith that is proportional to $d^{-2}$ with $d$ the diameter of the pebble. Note that the exponent of this distribution is higher than the current estimation post-Hayabusa mission that sets the exponent to -2.8 (Miyamoto et al. , 2007). Different pebbles sizes were considered in the range $[0.0002,0.2] \mathrm{m}$ with an average density of $2500 \mathrm{~kg} / \mathrm{m}^{3}$. Note that a higher size would result in a higher mass but a lower ejection velocity.

The spacecraft is assumed to be equipped with a stuffed whipple shield made of intermediate fabric layers (such as Nextel ceramic fibre or Kevlar aramid fibre) between an outer aluminium bumper plate and an inner aluminium wall (or rear wall) (Ryan and Christiansen , 2009). The distance between the bumper and the rear walls is $5 \mathrm{~cm}$. Both walls are made of Aluminium 7075-T6 with a tensile strength of $\sigma=78 \mathrm{ksi}$ and a density of $\rho_{b}=2.7 \mathrm{~g} / \mathrm{cm}^{3}$. For a pebble with diameter $d$, velocity $v_{p}$, mass $m_{p}$ and density $\rho_{p}$, hitting at an incident angle $\theta$, the thickness of the front wall is given by (Ryan and Christiansen , 2009):

$$
\tau_{b}=\frac{0.15 d \rho_{p}}{\rho_{b}}
$$

and the thickness of the rear wall by:

$$
\tau_{w}= \begin{cases}c_{w}\left(\frac{A D}{c_{0} d \rho_{p}}\right)^{-1.1} \frac{m_{p} v_{p} \cos \theta^{1.5}}{\rho_{w} S^{2}\left(\frac{\sigma}{40}\right)^{1 / 2}} & v_{p}>6.5 \mathrm{~km} / \mathrm{s} \\ \left(\frac{d \cos \theta^{4 / 3} v_{p}^{2 / 3} \rho_{p}}{2.35}-0.37 A D\right)\left(\frac{40}{\sigma}\right)^{1 / 2} & v_{p}<2.6 \mathrm{~km} / \mathrm{s}\end{cases}
$$


with $S$ the distance between the walls, $\rho_{w}=\rho_{b}, c_{w}=8.8, c_{0}=0.38, c_{s}=0.23$, and $A D$ given by:

$$
A D=\tau_{b} \rho_{b}+c_{s} d \rho_{p}
$$

Formulae (36) are valid for velocities of the pebble above $6.5 \mathrm{~km} / \mathrm{s}$ and below $2.6 \mathrm{~km} / \mathrm{s}$. For all velocity values of the pebble between 2.6 and $6.5 \mathrm{~km} / \mathrm{s}$, we used a linear interpolation with respect to the velocity $v_{p}$. For more details on shielding against debris and meteoroids refer to Ryan and Christiansen (2009). The assumption is that the normal to the front shield can be inclined by 15 degrees with respect to the incoming particles.

Figure 17 shows the thickness of the rear shield for different dimensions of the pebbles and corresponding intended $\delta v$, while Figure 18 shows the corresponding velocity of the pebbles. From these two figures it emerges that for small and fast projectiles even a thin shield is sufficient. For larger particles and slower velocities, a thicker shield is required. In both cases, however, the smaller the $\delta v$, the smaller the shield, down to the point in which the thickness of the rear wall becomes negative. For these combinations of diameter and $\delta v$, either a shield with a smaller distance between the walls or a simpler single-wall shield are sufficient.

\subsection{Transient-radiation effects on electronics}

In the examples presented above, the carrier spacecraft is located between $150 \mathrm{~km}$ and $300 \mathrm{~km}$ from the asteroid. Therefore, one could consider the effect of radiation on electronics as potential show stopper. Transient-radiation effects on electronics (TREE), as defined in Glasstone \& Dolan (1977), are not possible as such an event would require either a direct exposure to the radiation wave, or that the radiation wave penetrates through the asteroid without being absorbed.

An electromagnetic pulse (EMP) due to the interaction between the plasma and the local magnetic field would also be much smaller than on Earth. In fact, the only relevant magnetic field is the one generated by the Sun, that at $1 \mathrm{AU}$ is $10^{-9}$ tesla. Following a cubic law, the intensity at perihelion of the orbit selected for the example in this paper would be $2.4 \times 10^{-9}$ tesla and at aphelion $7.5 \times 10^{-10}$ tesla. By comparison, the magnetic field on the Earth varies between $[2.5,6.5] \times 10^{-5}$ tesla. This means that the EMP experienced by the onboard electronics would be four to five orders of magnitude weaker than on the Earth, assuming the asteroid is not providing any dissipation.

Note that, according to the model presented in this paper, the material vaporized during the explosion moves in a direction opposite to the mother 


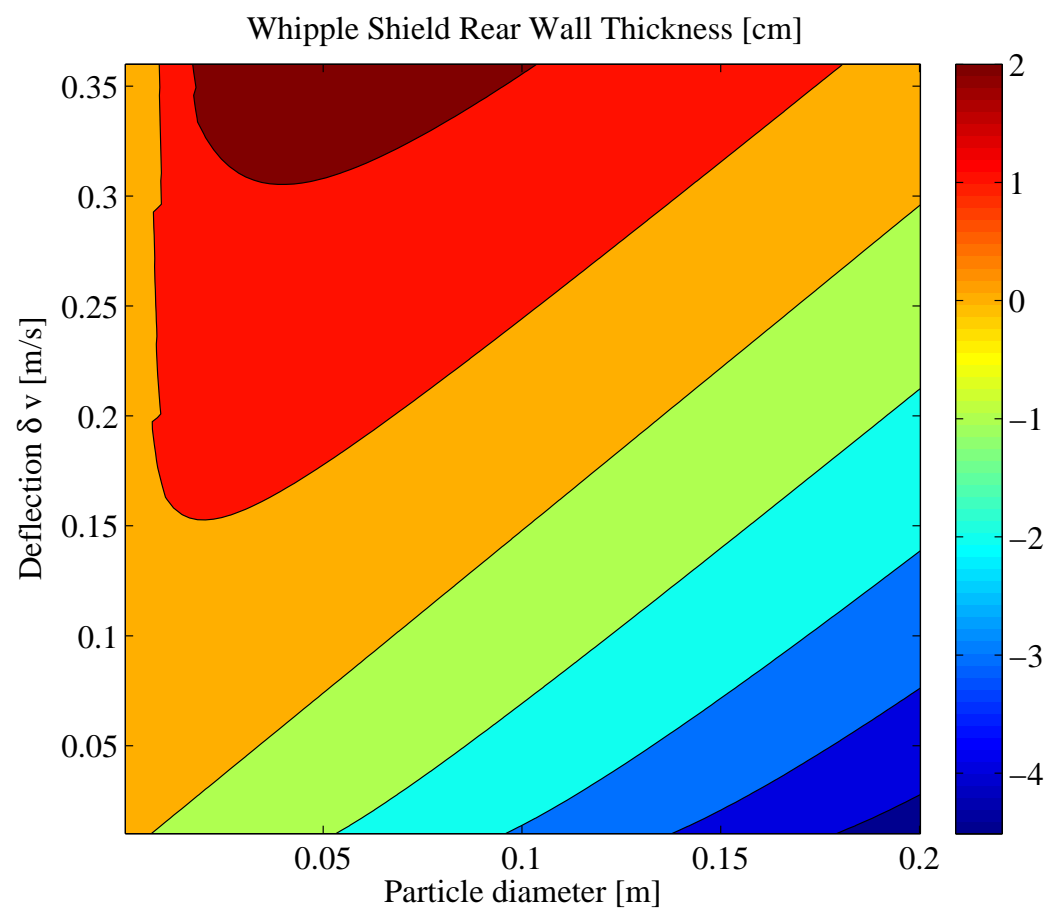

Figure 17: Thickness of the rear wall of the whipple shield as a function of particle size and expected deflection $\delta v$

spacecraft. The incident radiation cannot generate ionised material on the antipodal hemisphere due to the penetration depth of the radiation. On the other hand, the material on the surface that is not ejected will continue to evaporate after the explosion, potentially ejecting some ionised gas that can eventually reach the mother spacecraft. In this case we need to assume that the cooling of the material is slow or there is another constant source of heat, for example an activated radioactive layer of rocks, and significant vaporization continues even when the explosion crater is facing the spacecraft or that some of the vaporized material follows a trajectory that eventually hits the mother spacecraft.

We can then study the limit case in which significant ejection of ionised gas occurs in the direction of the mother spacecraft. Given $\rho$ the density of a layer of radioactive material, $h$ the thickness of this layer and $R$ the radioactive decay of the material in this layer (in $W / \mathrm{kg}$ ), the following an energy balance can be used to estimate the self-sustained vaporisation process 


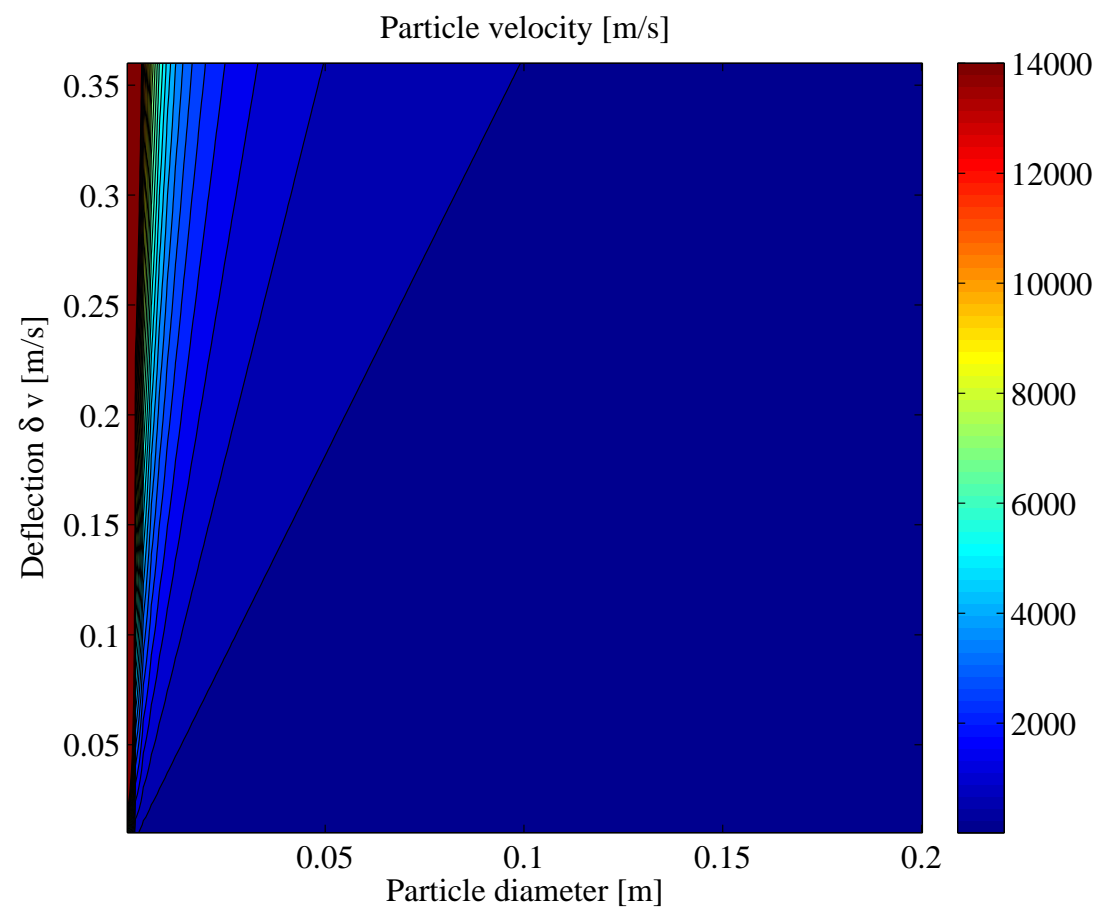

Figure 18: Particle velocity as a function of diameter and expected deflection $\delta v$

occurring post-explosion:

$$
\rho h R=\dot{\mu} E_{v}^{*}+\sigma \epsilon\left(T_{s}^{4}-T_{\infty}^{4}\right)
$$

In this expression, $E_{v}^{*}$ is an augmented enthalpy and $\dot{\mu}$ the mass flow rate, in $\mathrm{kg} / \mathrm{m}^{2} \mathrm{~s}$, at the surface of the asteroid, which is linked with the surface temperature through the Langmuir equation. Equation (39) computes the steady surface temperature $T_{s}$ from a given layer $h$ of radioactive material as well as the flow rate of particle evaporating from the surface of the asteroid. The flow rate is assumed to decrease at a rate relative to the inverse square of the distance.

For hovering distances of $200 \mathrm{~km}$ and $2000 \mathrm{~km}$, model (39) gives a density of particles that is lower than the one of the ionosphere as long as the parameter $(\rho h R)$ remains less than $10 \mathrm{MW} / \mathrm{m}^{2}$ (see Figure 19). A $250 \mathrm{~m}$ thick layer of Plutonium, with a radioactive decay of $2 \mathrm{~W} / \mathrm{kg}$, would barely be sufficient to approach such radiation levels. Additionally, due to the shielding, this flow would not distribute over the whole spacecraft but would mainly 
be concentrated on the shield. Given that a number of spacecraft survive in LEO for a long time ${ }^{4}$ we can safely conclude that this flow of ionised material is not a critical factor for the viability of the mission.

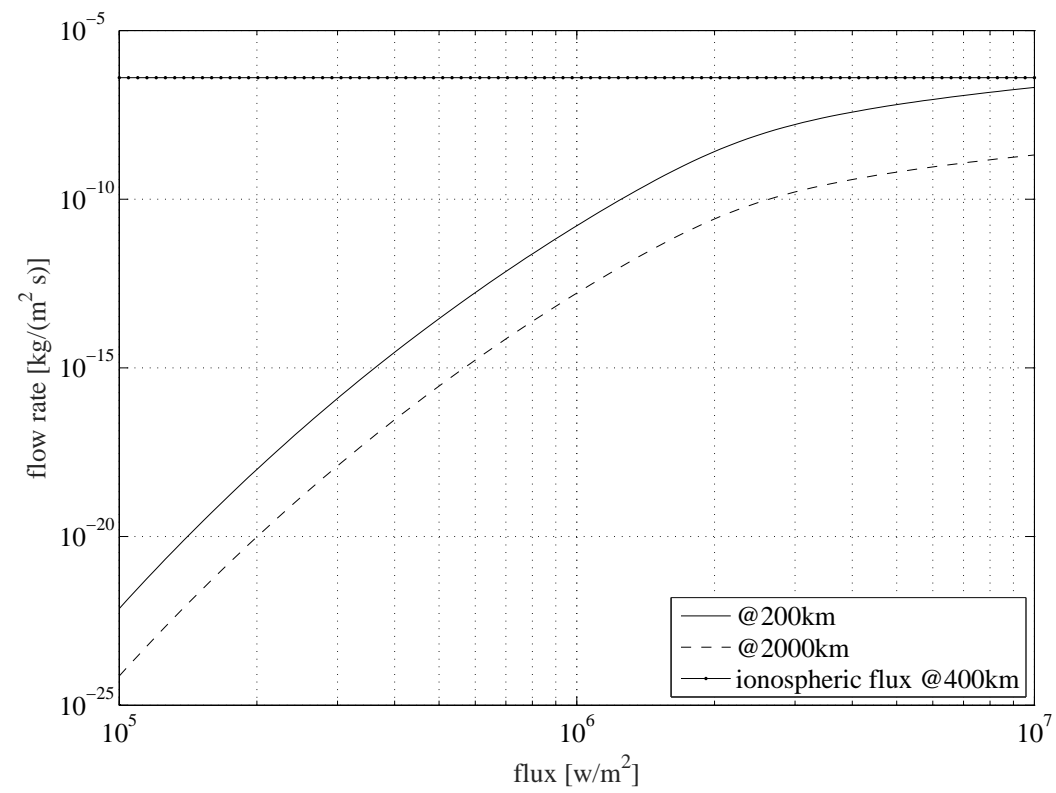

Figure 19: Flux of ionised particles at different distances from the asteroid as a function of the required surface heat power density to sustain vaporisation

\subsection{Post-explosion radiation exposure}

It can be argued that some of the activated material remains on the surface of the asteroid or evaporates during the slow evaporation process described by Eq. (39). The estimation of the total dose received by the spacecraft is not an easy calculation and is out of the scope of this paper. One can consider that all constituent materials of an S-type asteroid that can be activated during an explosion have a very short decay time in general and all decay emitting $\beta$ or $\alpha$ particles. For example, silica has isotopes with a decay time that ranges from 60 ns for $S i_{14}^{43}$ to 157 minutes for $S i_{14}^{31}$ and decay with the emission of $\beta+$ or $\beta$ - particles. Only $S i_{14}^{32}$ decays in 153 years but still with $\beta$ - radiations

\footnotetext{
${ }^{4}$ See the European Space Agency GOCE spacecraft as an example, www.esa.int/Our_Activities/Observing_the_Earth/GOCE.
} 
IAEA (2014). High energy neutrons can only be spontaneously generated by the decay of heavy atoms that cannot come from the core of the asteroid post explosion (Litz et al. , 2012). Neutron activation on the antipodal side is also not possible, neither neutron scattering. Neutrons can be produced as secondary radiation when alpha particles interact with the walls of the spacecraft. The same happens in general with galactic cosmic rays and solar energetic particles.

As the products of the explosion are ejected with the layer of ablated material, they cannot provide heavy atoms. The real source of radiations post-burst is the layer of rocks that are not ejected. A correct evaluation of the total radiation dose post explosion is case-dependent, but the low flux of ionised material will correspond to an equally low flux of alpha and beta particles due to the decay of the isotopes in the flow of gas. The proposed Whipple shield can help to mitigate the risk of a failure due to this flow of radiation, as for example Kevlar Aramid are polymers with a good content of hydrogen. In addition, as for the debris flux, one can place the spacecraft at a greater distance to significantly reduce the total dose. Therefore, a proper combination of distance and thickness of the shield can mitigate the risk of a failure due to post explosion radiation exposure.

\subsection{Failure Analysis}

We now analyse the consequence of a partial or total failure of the cycler after each explosion. The worst case scenario is that no bomb can be exploded after a failure occurs. As explained previously, the higher the number of bombs, the lower the failure probability per explosion because the yield is lower and all the possible sources of damage to the mother spacecraft are proportionally reduced.

The follow analysis looks as the case of six explosions, computing the miss distance for different failure occurrences, for example if the carrier spacecraft fails completely after the first, second or third explosion. The result is represented in Figure 20.

\section{Conclusion and Future Works}

This paper proposed a novel deflection method, called the nuclear cycler concept, derived from the nuclear interceptor concept. This incremental strategy bridges the gap between traditional impulsive and slow-push methods by combining the advantages of the single nuclear interceptor method, 


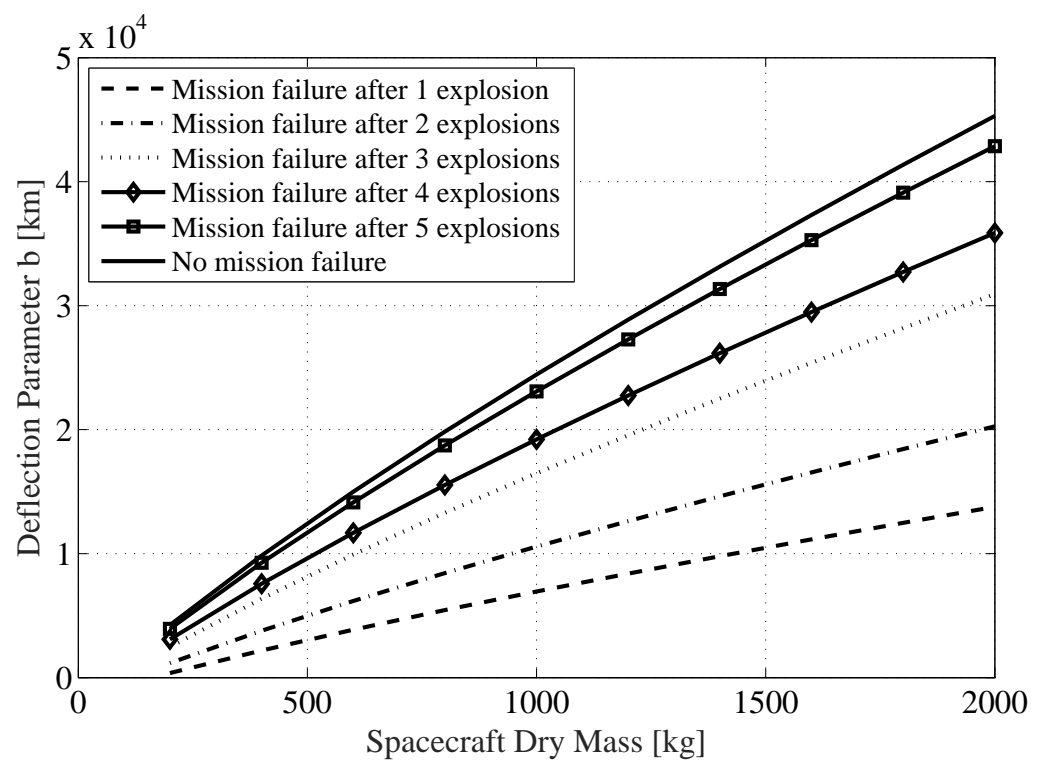

Figure 20: Deflection parameter for different failure occurrences

which is often quoted as the most effective way of deflecting an asteroid, with the superior controllability offered by slow push methods. The nuclear cycler approach could be used to precisely manipulate the trajectory of an asteroid with a high degree of redundancy, something not feasible by a single impulsive strategy. In addition, during a given cycle, the data generated by the past explosions can be analysed in order to improve the efficiency of the next cycle. The analyses in this paper were limited to the case in which explosions occur at the apsidal points. More frequent explosions are possible but this analysis is left for future studies. Further analyses are also required in order to have a full picture regarding the range of applicability of the nuclear cycler method and to assess the impact of the different parameters contributing to the effectiveness of this method.

\section{Acknowledgement}

This work is funded by the European Commission Framework Programme 7, through the Stardust Marie Curie Initial Training Network (www.stardust2013.eu), FP7-PEOPLE-2012-ITN, Grant Agreement 317185. 


\section{References}

Adams, J. H. Jr., Hathaway, D. H., Grugel, R. N., Watts, J.W., Parnell, T.A. Gregory, J.C., Winglee, R.M., Revolutionary Concepts of Radiation Shielding for Human Exploration of Space. NASA/TM?2005?213688, March 2005.

Bombardelli, C., Pel?ez, J., Ion Beam Shepherd for Asteroid Deflection, Journal of Guidance, Control and Dynamics, Vol. 34, No. 4, pp. 1270-1272, 2011

Glasstone, S., The Effects of Nuclear Weapons, U.S. Atomic Energy Commission, 1962.

Glasstone,S. and Dolan,P. J.. The Effects of Nuclear Weapons, Third Edition. U.S. Government Printing Office. Washington, D.C., 1977.

Hammerling, P., Remo, J. L., NEO Interaction with Nuclear Radiation, Acta Astronautica, Vol. 36, No. 6, pp. 337-346, 1995

Hu, W., Scheeres, D J, Spacecraft Motion About Slowly Rotating Asteroids, Journal of Guidance, Control and Dynamics, Vol. 25, No. 4, pp. 765-775, 2002

International Atomic Energy Agency, Nuclear Data Service. https : //wwwnds.iaea.org/

Jutzia, M., Michel, P. Hypervelocity impacts on asteroids and momentum transfer I. Numerical simulations using porous targets, Icarus, Vol. 229, pp. 247-253, 2014

Litz, M., Waits, c., Mullins, J., Neutron-Activated Gamma-Emission: Technology Review, Army Research Laboratory,ARL-TR-5871, Adelphi, MD 20783-1197, January 2012.

Lu, E. T., Love, S. G., Gravitational tractor for towing asteroids, Nature, Vol. 438, pp. 177-178, 2005

Martin,R., Komatitsch, D., Blitz,C., Le Goff,N., Simulation of Seismic Wave Propagation in an Asteroid Based upon an Unstructured MPI SpectralElement Method: Blocking and Non-blocking Communication Strategies, 
High Performance Computing for Computational Science ? 1 VECPAR 2008, 8th International Conference Toulouse, France, June 2008, Lecture Notes in Computer Science 5336.

Meshcheryakov, S., D7.3 Assessment of blast deflection and other mitigation concepts, Report of the NEOShield, A Global Approach to Near-Earth Object Impact Threat Mitigation, FP7-SPACE-2011-282703, 15 April 2014.

Meshcheryakov S. A. and Lipnitskii Yu. M.. Estimated Efficiency of the Deflection of a Dangerous Space Object Using an Explosion or Impact. Technical Physics. Vol. 60, No. 1, pp. 26-30. , 2015

Miyamoto.H., Yano,H., Scheeres,D., J. , Abe,S., Barnouin-Jha,O., Cheng, A., F., Demura,H., Gaskell,R.,W., Hirata,N., Ishiguro,M., Michikami,T., Nakamura,A., M., Nakamura,R., Saito,J., Sasaki,S., Regolith Migration and Sorting on Asteroid Itokawa, Science, Vol. 316, No. 18, May 2007.

NASA, Near-Earth Object Survey and Deflection Analysis of Alternatives, Report to congress, 2007.

Olds, J., Charania, A., Graham, M., Wallace, J., The League of Extraordinary Machines: A Rapid and Scalable Approach to Planetary Defense Against Asteroid Impactors, NASA Institute for Advanced Concepts, CPNIAC 02-02, Vol. 1, 2004

Plesko, C. S., Weaver, R. P., Huebner, W. F., Numerical and Probabilistic Analysis of Asteroid and Comet Impact Hazard Mitigation. Proceedings of the Advanced Maui Optical and Space Surveillance Technologies Conference, Hawaii, 2010.

Rossi, A., Marzari, F., Farinella, P., Orbital Evolution Around Irregular Bodies, Earth, Planets, Space, 1999, Vol. 51, pp. 1173-1180

Ryan,S., Christiansen, E.,L., Micrometeoroid and Orbital Debris (MMOD) Shield Ballistic Limit Analysis Program, NASA/TM?2009?214789, February 2010.

Sanchez Cuartielles, J. P., Colombo, C., Vasile, M., \& Radice G., Multicriteria comparison among several mitigation strategies for dangerous Near Earth Objects, Journal of Guidance, Control and Dynamics, Vol. 32, pp. 121-42, 2009 
Sanchez Cuartielles, J. P., Vasile, M., Radice, G. Consequences of asteroid fragmentation during impact hazard mitigation, Journal of Guidance, Control and Dynamics, 33, No. 1, pp. 126-146, 2010

Sazonov, Yakovlev, Explosion method of preventing collisions of asteroidcomet bodies with the Earth in the case of their late detection. Journal of Engineering Physics and Thermophysics, Volume 79, Issue 3, pp. 476-488, 2006

Schaub, H., Junkins, J. L., Analytical mechanics of space systems, AIAA Education Series, Virginia, USA, 2003

Shubin, O.,N., Nechai,V.,Z., Nogin,V.,N., Petrov,D.,V., Simonenko,V.,A., Nuclear Explosion Near Surface of Astroids and Comets, Common Description of the Phenomenon. Proceedings of the Planetary Defense Workshop, 1995, Livermore, USA.

Solem, J. C., Interception of comets and asteroids on collision course with Earth, Journal of Spacecraft and Rockets, Vol. 30, No. 2 (1993), pp. 222228.

Tedeschi, W.J., Remo, J.L., Schulze, J.F., Young, R.P., Experimental hypervelocity impact effects on simulated planetesimal materials, International Journal of Impact Engineering, Volume 17, Issues 4-6, pp. 837-848, 1995

Vasile, M. and Colombo, C., Optimal Impact Strategies for Asteroid Deflection, Journal of Guidance, Control and Dynamics, Vol. 31, No 4, pp. 858-873, 2008.

Vasile, M. and Maddock, C., Design of a formation of solar pumped lasers for asteroid deflection, Advances in Space Research, Vol. 50, Issue 7, pp. 891-905, 2012

Wie, B., Dynamics and Control of Gravity Tractor Spacecraft for Asteroid Deflection, Journal of Guidance, Control and Dynamics, Vol. 33, No. 5, pp. 1413-1423, 2008 